\title{
Alternative transmission patterns in independently acquired nutritional co-symbionts of Dictyopharidae planthoppers
}

\author{
Anna Michalik ${ }^{*}$, Diego C. Franco ${ }^{2}$, Michał Kobiałka ${ }^{1}$, Teresa Szklarzewicz ${ }^{1}$, Adam Stroiński ${ }^{3}$, \\ Piotr Łukasik ${ }^{2}$
}

${ }^{1}$ Department of Developmental Biology and Morphology of Invertebrates, Institute of Zoology and Biomedical Research, Faculty of Biology, Jagiellonian University, Gronostajowa 9, 30-387 Kraków, Poland

${ }_{2}^{2}$ Institute of Environmental Sciences, Faculty of Biology, Jagiellonian University, Gronostajowa 7, 30-387 Kraków, Poland

${ }^{3}$ Museum and Institute of Zoology, Polish Academy of Sciences, Wilcza 64, 00-679 Warszawa, Poland

\begin{abstract}
Sap-sucking hemipterans host specialized, heritable microorganisms that supplement their unbalanced diet with essential nutrients. These microbes show unusual features that provide a unique perspective on the evolution of life but have not been systematically studied. Here, we combine microscopy with high-throughput sequencing to revisit 80 year-old reports on the diversity of symbiont transmission modes in a broadly distributed planthopper family Dictyopharidae. We show that in all species examined, the ancestral nutritional endosymbionts Sulcia and Vidania are complemented by co-primary symbionts, either Arsenophonus or Sodalis, acquired several times independently by different host lineages. Like in other obligate sap-feeders, the ancestral symbionts produce essential amino acids, whereas co-primary symbionts contribute to the biosynthesis of $\mathrm{B}$ vitamins. These symbionts reside within separate bacteriomes within the abdominal cavity, although in females, Vidania also occupies bacteriocytes in the rectal organ. Notably, the symbionts are transmitted from mothers to offspring in two alternative ways. In most examined species, all nutritional symbionts simultaneously infect the posterior end of the full-grown (vitellogenic) oocytes and next gather in their perivitelline space. In contrast, in other species, Sodalis colonizes the cytoplasm of the anterior pole of young (previtellogenic) oocytes forming a cluster separate from the "symbiont ball" formed by late-invading Sulcia and Vidania. Our data add to evidence on frequent replacements of gammaproteobacterial symbionts combined with the relative functional stability of the nutritional functions during the evolution of sap-feeding insects, and show how newly-arriving microbes may utilize different strategies to establish long-term heritable symbiosis.
\end{abstract}

Keywords: planthoppers, nutritional endosymbiosis, transovarial transmission

\section{Significance statement}

Sup-sucking hemipterans host ancient heritable microorganisms that supplement their unbalanced diet with essential nutrients, and which have repeatedly been complemented or replaced by other microorganisms. They need to be reliably transmitted to subsequent generations through the reproductive system, and often they end up using the same route as the ancient symbionts. We show for the first time that in a single family of planthoppers, the complementing symbionts that have established infections independently utilize different transmission strategies, one of them novel, with the transmission of different microbes separated spatially and temporarily. These data show how newly-arriving microbes may utilize different strategies to establish long-term heritable symbiosis.

\footnotetext{
* corresponding authors: Anna Michalik

E-mail: a.michalik@uj.edu.pl
}

Author Contributions: $\mathrm{AM}$ and $\mathrm{P} \mathrm{L}$ designed research, AS conducted the sampling, AM, DCF, MK, TS and PŁ performed research and analyzed data, AM, DCF and PL wrote the paper.

Competing Interest Statement: The authors declare no conflict of interests

Classification: Biological Sciences: Cell biology, Developmental Biology, Evolution, Developmental Biology 

made available under aCC-BY 4.0 International license.

\section{Introduction}

Mutualistic relationships with heritable bacterial and/or fungal microorganisms have played crucial roles in the biology of multiple groups of insects, contributing significantly to their evolutionary and ecological success (13 ). The growing awareness of the diversity and importance of insect symbioses, in addition to the rapid development in sequencing-based techniques, has led to an increased interest in these associations. However, outside a few model species and some reasonably well-sampled clades, our knowledge of the diversity, evolution, and biological characteristics of the microbial symbionts, and the microbial roles in the evolution of insect diversity remains limited $(4,5)$. Among insects, sapsucking hemipterans are obligatorily dependent on heritable nutritional microbes that supplement their unbalanced diet with essential amino acids, vitamins, and co-factors (4, 6-8). Multiple symbiont combinations have been described from Auchenorrhyncha, a suborder comprising infraorders Fulgoromorpha - planthoppers, and Cicadomorpha cicadas, spittlebugs, treehoppers, and leafhoppers $(4,9)$. Their common ancestor that lived about 300 million years ago (MYA) is thought to have became colonized by two microbes, a Bacteroidetes currently known as Candidatus Sulcia muelleri (further referred to as Sulcia) and a beta proteobacterium, variably known as Candidatus Nasuia deltocephalinicola, Candidatus Zinderia insecticola or Candidatus Vidania fulgoroidaea (further referred to as Nasuia, Zinderia, and Vidania, respectively, or as betasymbionts collectively) (9-12). These nutrient-providing symbionts have become obligate components of the host biology, and transmitting strictly maternally, they have codiversified with hosts. However, in many host clades, one or both became complemented or replaced by other microbes. For example, an Alphaproteobacterium Candidatus Hodgkinia cicadicola (further referred to as Hodgkinia) has replaced the beta-symbiont in the ancestor of modern cicadas (family Cicadidae), but has itself became repeatedly replaced by fungi from the genus Ophiocordyceps $(13,14)$.

In these multi-partite symbioses, microbes share the responsibility for essential nutrient biosynthesis. For example, in known Cicadomorpha, Sulcia encodes pathways for producing 7 or 8 essential amino acids, whereas the remaining 3 or 2 amino acids are provided by their symbiotic partner $(8,9,12)$. The situation can get more complicated when one of the ancient microbes gets replaced by another, or when the host is colonized by more than two symbionts and the nutritional functions become subdivided among a greater number of partners. This has occurred repeatedly in different lineages of Auchenorrhyncha, where additional symbionts have either taken over beta-symbiont's role or contribute to vitamin biosynthesis (4).

These intimate and intricate metabolic interdependencies between insects and associated microorganisms indicate a vital role of nutritional symbionts in host biology, and the need to ensure reliable transmission of these essential partners across generations. In sap-feeding hemipterans, transovarial transmission through female germ cells predominates (15-17). It may take place at all stages of female germ cell development. In some insects, symbionts infect undifferentiated germ cells, or alternatively, young, previtellogenic oocytes (17). However, in most hemipteran taxa, including all Auchenorrhyncha studied to date, they invade ovarioles containing older (vitellogenic or choriogenic) oocytes. The complementation or replacement of ancient, co-adapted heritable nutritional symbionts by newly arriving microbes, while likely beneficial to the hosts because of their greater metabolic capacity and efficiency, creates apparent challenges for their transmission. Host lineages that acquired new symbionts have to develop new traits and mechanisms of their effective vertical transmission, which is crucial for the fixation of new symbiosis (18), being also a matter of life or death to the newly established symbionts. The evolution of the symbiont transmission and the symbiont replacements are inseparably linked, and we need to study one to understand another.

Buchner (15) while summarizing decades of microscopy-based research on Auchenorrhyncha symbioses, famously wrote about "the veritable fairyland of insect symbiosis", apparently referring to the diversity of microbes he observed in different host clades, as well as their transmission mechanisms. However, he lacked tools to fully characterize the evolution of symbioses across the auchenorrhynchan phylogeny. The popularization of DNA sequencing-based techniques enabled such investigation, but surprisingly, our knowledge of these links remains limited to a few Auchenorrhyncha clades, mainly within Cicadomorpha (12, 18, 19). While diagnostic screens revealed Sulcia, Vidania, and often other bacteria or fungi in most planthopper families, in only one planthopper species so far nutritional symbionts have been characterized using genomics. In the Hawaiian cixiid Oliarus filicicola, Vidania produces seven essential amino acids, Sulcia synthesizes three, whereas a more recently acquired gammaproteobacterial symbiont Purcelliella contributes Bvitamins. This rearrangement of Sulcia and Vidania nutritional responsibilities shed new light on the evolution of planthopper symbioses and how they can be influenced by infections with additional microbes. However our understanding of symbioses in this diverse, widespread, and ecologically significant insect clade remains very limited.

This project aimed to provide an insight into the diversity and biology of symbioses in the large, diverse planthopper family Dictyopharidae. Eight members of this family were shown to possess bacteria Vidania and usually also Sulcia (20), but microscopic observations conducted by Müller $(21,22)$ and summarized by Buchner (15) indicated that Dictyopharidae also host the third symbiont. 
bioRxiv preprint doi: https://doi.org/10.1101/2021.04.07.438848; this version posted April 9, 2021. The copyright holder for this preprint (which was not certified by peer review) is the author/funder, who has granted bioRxiv a license to display the preprint in perpetuity. It is made available under aCC-BY 4.0 International license.

Strikingly, they reported that in different Dictyopharidae species, these additional symbionts can be transmitted in alternative ways, either together or separately from Sulcia and Vidania. While this suggested the independent origins of the additional symbionts, or other unusual biological or evolutionary processes, Buchner and colleagues lacked the tools to fully describe and explain the phenomenon. There was, however, little doubt that Dictyopharidae can provide unique insights into the symbiont evolution, transmission, and replacements, making this family a valuable model group for the exploration of symbiont complementation and genome evolution.

Here, we report the results of microscopy and sequencing-based investigations of symbiotic bacteria associated with seven species belonging to the Dictyopharidae. We survey the diversity of the symbionts and explore their nutritional roles. In particular, we focus on how symbionts in this group are transmitted across generations. We describe and discuss how the auchenorrhynchan symbiont transmission can be separated in time and space, how this may have evolved, and what it may mean for the host.

\section{Results}

Dictyopharidae planthoppers harbor (at least) three types of nutritional symbionts

Analyzes of marker gene sequences for the representative specimens of seven experimental species confirmed their morphology-based identifications (Fig. 1A, SI Appendix, Figs. S3, and S4). The phylogeny revealed two well-supported clades corresponding to subfamilies Dictyopharinae and Orgerinae; it also showed that $R$. edirneus is more closely related to $P$. platypus than to $R$. scytha, in agreement with the proposed taxonomic revision of the group (Emeljanow, 2003). We successfully amplified bacterial 16S rRNA gene V4 region from the abdomens of all experimental individuals from these seven Dictyopharidae species. The total number of $16 \mathrm{~S}$ rRNA reads passed through all analysis steps was 452,949 , or 23,840 per sample on average (Dataset Table S5). Clustering with 97\% identity cutoff identified 67 OTUs.
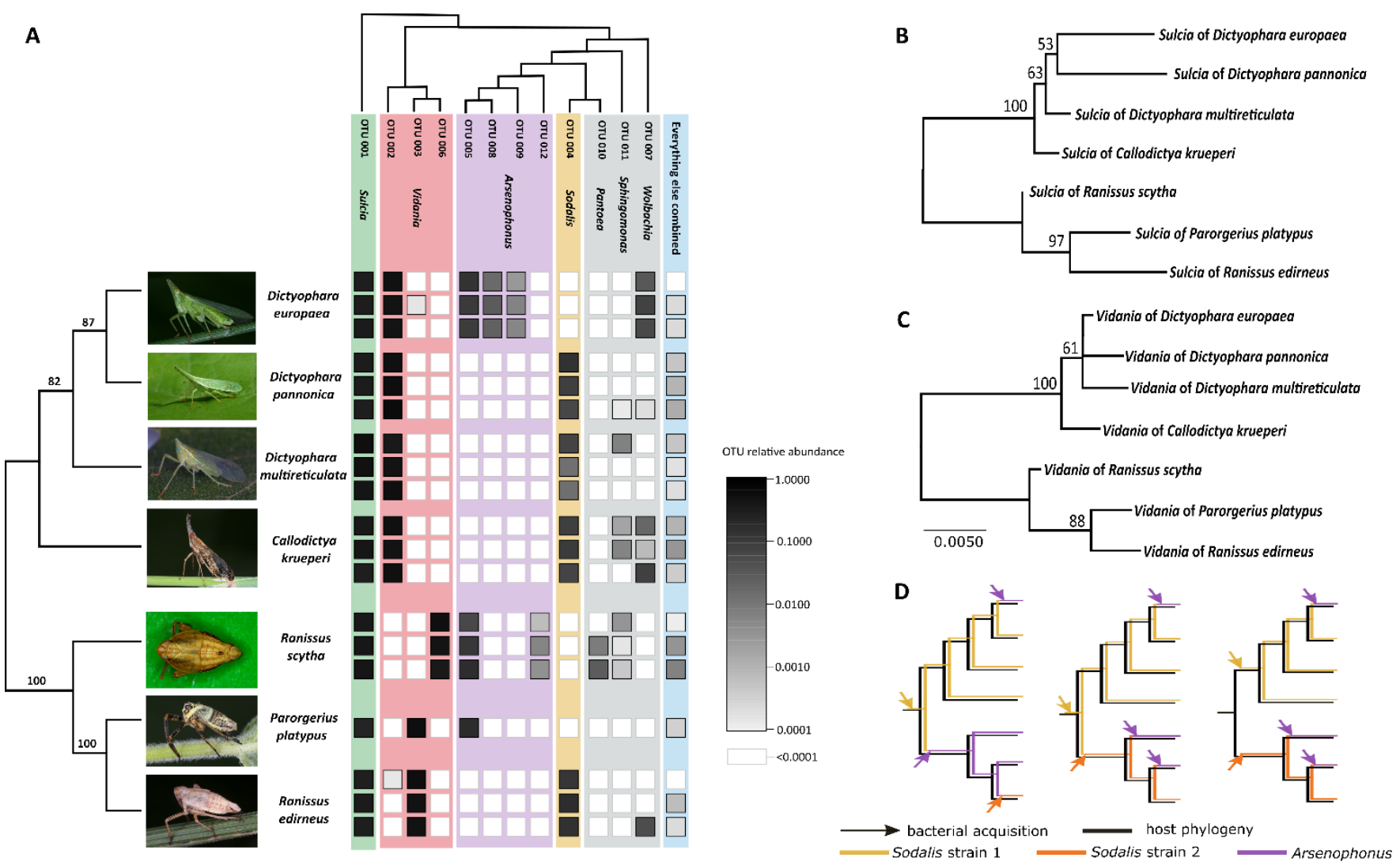

Figure 1. The bacterial communities in Dictyopharidae planthoppers. A. The diversity and relative abundance of bacteria in replicate specimens from seven experimental species. Insect phylogeny (Maximum Likelihood) is based on concatenated marker gene sequences (COI, CytB, 18S rRNA, and 28S rRNA); bootstrap values are shown above the nodes. The bacterial tree is based on representative sequences of 16S rRNA V4 region for different OTUs. B \& C. ML phylogenies for Sulcia and Vidania symbionts from seven experimental species, based on full-length 16S rRNA sequences. D. Three of many possible scenarios of Sodalis/ Arsenophonus acquisition and replacement during Dictyopharidae diversification. 
All studied individuals hosted Sulcia and Vidania, and additionally, either Sodalis or Arsenophonus (Fig. 1A, SI Appendix, Fig. S1, Dataset Table S5). Together, these symbionts comprised $>97 \%$ of reads in each of the libraries. The single-nucleotide-resolution data (Dataset Table S6) for these dominant symbionts revealed that all species harbor very similar strains of the slow-evolving symbiont Sulcia, all clustering to a single 97\% OTU. In contrast, Vidania genotypes from different species were sufficiently divergent to fall into three distinct $97 \%$ OTUs. C. krueperi, D. multireticulata, D. pannonica, and $R$. edirneus hosted different genotypes of Sodalis (clustering to the same OTU). D. europaea, S. scytha and P. platypus hosted Arsenophonus, typically different genotypes from more than one OTU. However, their consistent relative abundance in replicate individuals strongly suggests that these genotypes and OTUs correspond to different rRNA operons within the genome of a single Arsenophonus strain (Fig. 1A). Less abundant microbial OTUs present in some species included Wolbachia, Pantoea, and Sphingomonas (Fig. 1A). All the remaining OTUs combined accounted for $0.2 \%$ of the total number of reads, and many of them represented contaminants or symbiont-derived sequences that accumulated large numbers of errors. We did not consider them further.

Phylogenetic trees of Sulcia and Vidania based on full-length 16S rRNA gene sequences (Fig. 1B, C) are congruent with the phylogeny of the seven experimental Dictyopharidae species (Fig. 1A), or a broader range of planthoppers (Suppl. Figs S2 and S3), as expected for symbionts co-diversifying with hosts. In contrast, $16 \mathrm{~S}$ rRNA gene phylogenies for Sodalis and Arsenophonus from diverse hosts, while poorly resolved and supported, were suggestive of independent origins of at least some of co-symbionts of Dictyopharidae (SI Appendix, Figs. S4 and S5). These data, combined with information on the distribution of the two symbiont clades across the host phylogeny and differences in transmission patterns and genomics characteristics (described later), are suggestive of several independent infections by Arsenophonus/Sodalis, or their repeated replacements by other strains of these symbionts (Fig 1B, C, D, SI Appendix, Figs 2-5). Unfortunately, the currently available data do not allow for a reconstruction of the order of these infections; we lack the resolution to distinguish among the many possible scenarios.

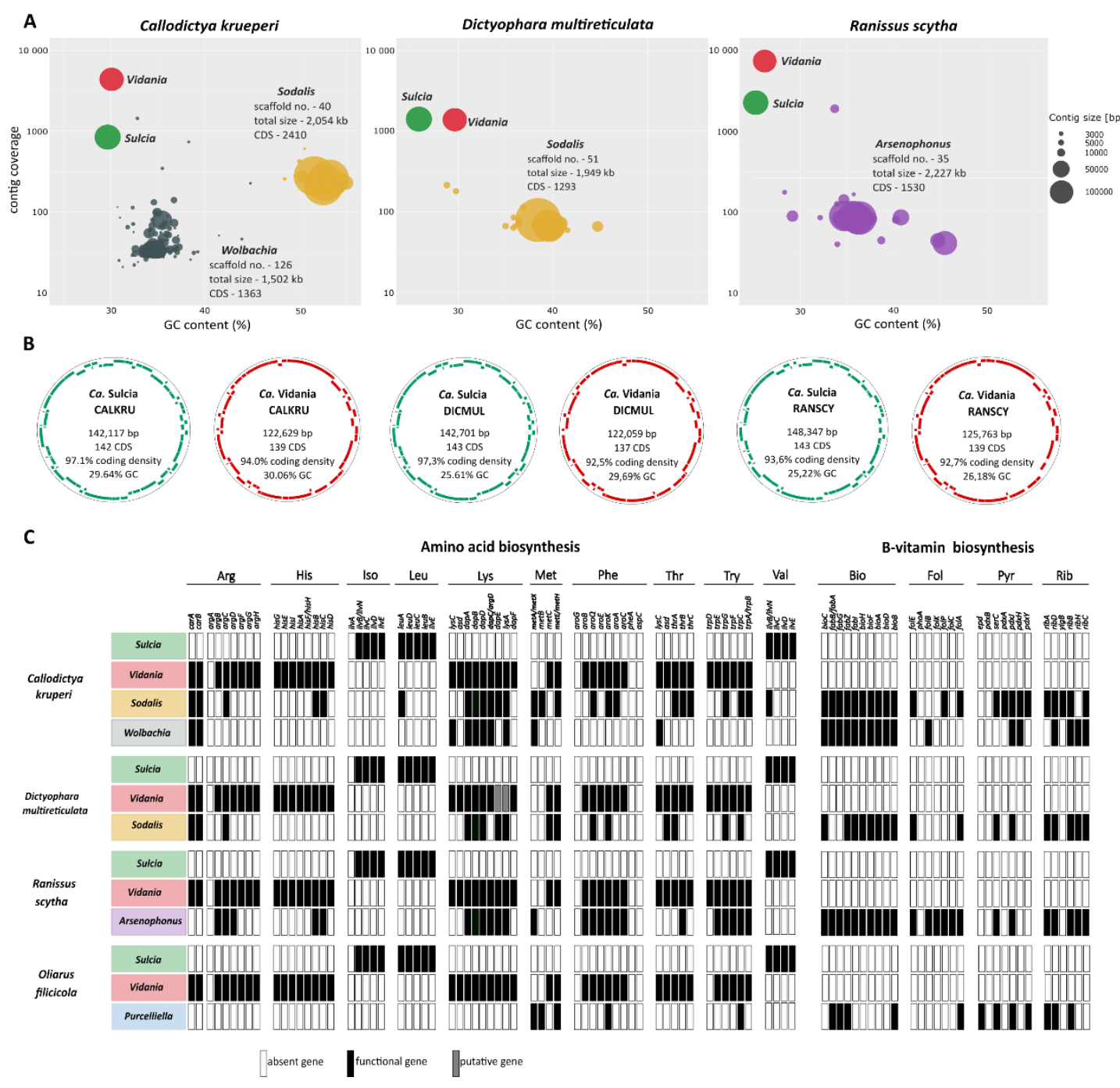


Figure 2. The summary of metagenomic data for three Dictyopharidae species. A. Taxon-annotated GC contents-coverage plots for metagenomic assemblies. Colored blobs represent scaffolds corresponding to the identified symbiont genome fragments. B. Circular diagrams of Sulcia and Vidania genomes from three species, showing gene positions on forward and reverse strands, and basic genome characteristics. C. Contribution of symbionts to amino acid and B-vitamin biosynthesis pathways. Standard abbreviations for amino acid, B-vitamin, and gene names are used.

Three of these scenarios, all assuming relatively few replacement events and shared ancestry of some strains, are presented in Fig. 1D. However, the number of independent infections or replacements along the tree branches could have been much greater. In fact, patterns such as the $16 \mathrm{~S}$ rRNA amplicon genotype diversity across $D$. pannonica individuals (Dataset Table S7) and some morphological differences among Sodalis cell populations hint at the possibility of recent or perhaps ongoing replacements.

\section{Sulcia and Vidania provide essential amino acids to host insects, whereas Arsenophonus, Sodalis, and Wolbachia supplement their diet with B - vitamins}

The symbiont genomes identified in the assemblies matched the dominant OTUs identified by amplicon sequencing (Fig. 2A). All species hosted Sulcia and Vidania, and in all cases, we were able to assemble circular genomes with sizes ranging between 142-148 kb for Sulcia and 122$125 \mathrm{~kb}$ for Vidania, characterized by relatively low GC contents (25-30\%) and high coverage (800-10000x) (Fig. 2B). Sulcia and Vidania genomes were co-linear relative to each other and the slightly larger reference genomes of $O$. filicicola (OLIH) symbionts (157 and $136 \mathrm{~kb}$, respectively Bennett \& Mao 2018; Suppl. Figs S6 and S7). Our metagenomic analyses also confirmed the presence of Sodalis and Wolbachia in CALKRU, Sodalis in DICMUL, and Arsenophonus in RANSCY. The genomic assemblies of these symbionts were fragmented; the number of scaffolds ranged between 23-126 and their total size between $1500 \mathrm{~kb}$ and $2220 \mathrm{~kb}$. The scaffold read coverage was substantially lower than in the case of Sulcia/Vidania but also relatively variable, particularly in the case of Arsenophonus (Dataset Table S7).

In the terms of gene contents, the newly characterized Sulcia and Vidania genomes are very similar to each other and the previously described OLIH symbiont genomes (Bennett and Mao, 2018). Sulcia genomes encode 142-143 predicted protein-coding genes, 27-29 identifiable tRNAs, complete ribosomal operon, and their coding density ranges from $93.6 \%$ to $97.3 \%$. Vidania genomes encode 137 139 predicted protein-coding genes, 23-25 tRNAs, complete ribosomal operon and coding density ranging between 92.5\%-94\% (Fig. 2B). In the two analyzed Sodalis genomes, prokka identified 2410 (CALKRU) and 1132 (DICMUL) predicted protein-coding genes, in Arsenophonus 1530, and in Wolbachia, 1363. However, because of the incompleteness of the assemblies of these co-symbiont genomes and challenges with pseudogene annotation, these numbers are approximate.

Our analyses of ancestral symbionts Sulcia and Vidania protein-coding genes revealed that they complement each other to supplement host insects with essential amino acids, in a way consistent among the three Dictyopharidae and OLIH (Fig. 2C). Sulcia participates in the biosynthesis of three out of ten essential amino acids, including isoleucine, leucine, and valine, whereas Vidania is involved in synthesizing the remaining seven. Interestingly, some of the genes in amino acid biosynthetic pathways (isoleucine, arginine, methionine, phenylalanine) were not found in Sulcia and Vidania genomes (Fig. 2C). Some of these missing amino acid biosynthesis genes, and duplicate copies of some others, are present in Sodalis, Arsenophonus, and Wolbachia genomes. In C. krueperi, Vidania lacks the first two genes essential for methionine biosynthesis from homoserine; however, these missing genes are present in the Sodalis genome. Similarly, in D. multireticulata, Sodalis complements two pseudogenes (dapE, lysA) in the Vidania genome essential in the lysine biosynthesis pathway. Arsenophonus from $R$. scytha has the same set of genes from phenylalanine and tryptophan biosynthesis as Vidania. In all Dictyopharidae, the gammaproteobacterial symbionts encode many genes in the lysine biosynthesis pathway and scattered genes from other pathways. We can not rule out the presence in the third co-primary symbiont genomes' remaining genes missing in Sulcia and Vidania due to the uncompleted assembly process.

In addition to genes that might participate in amino acids' biosynthesis, Sodalis, Arsenophonus, and Wolbachia contribute to the synthesis of B-vitamins: biotin, folate, riboflavin, and pyridoxine (Fig. 2C). Sodalis and Wolbachia associated with $C$. krueperi as well as Arsenophonus of $R$. scytha encode complete sets of biotin biosynthesis genes, whereas the genome of Sodalis of D. multireticulata carries only 8 of 10 genes from that pathway. Arsenophonus symbiont genome also encodes almost all genes involved in the biosynthesis of folate and riboflavin. Interestingly, in $C$. krueperi, the riboflavin pathway is shared by Sodalis and Wolbachia.

\section{Nutritional symbionts of dictyopharids occupy distinct but adjacent bacteriocytes}

In all dictyopharids studied, we found nutritional symbionts (Sulcia, Vidania, and either Sodalis or Arsenophonus) contained within separate bacteriomes 

made available under aCC-BY 4.0 International license.

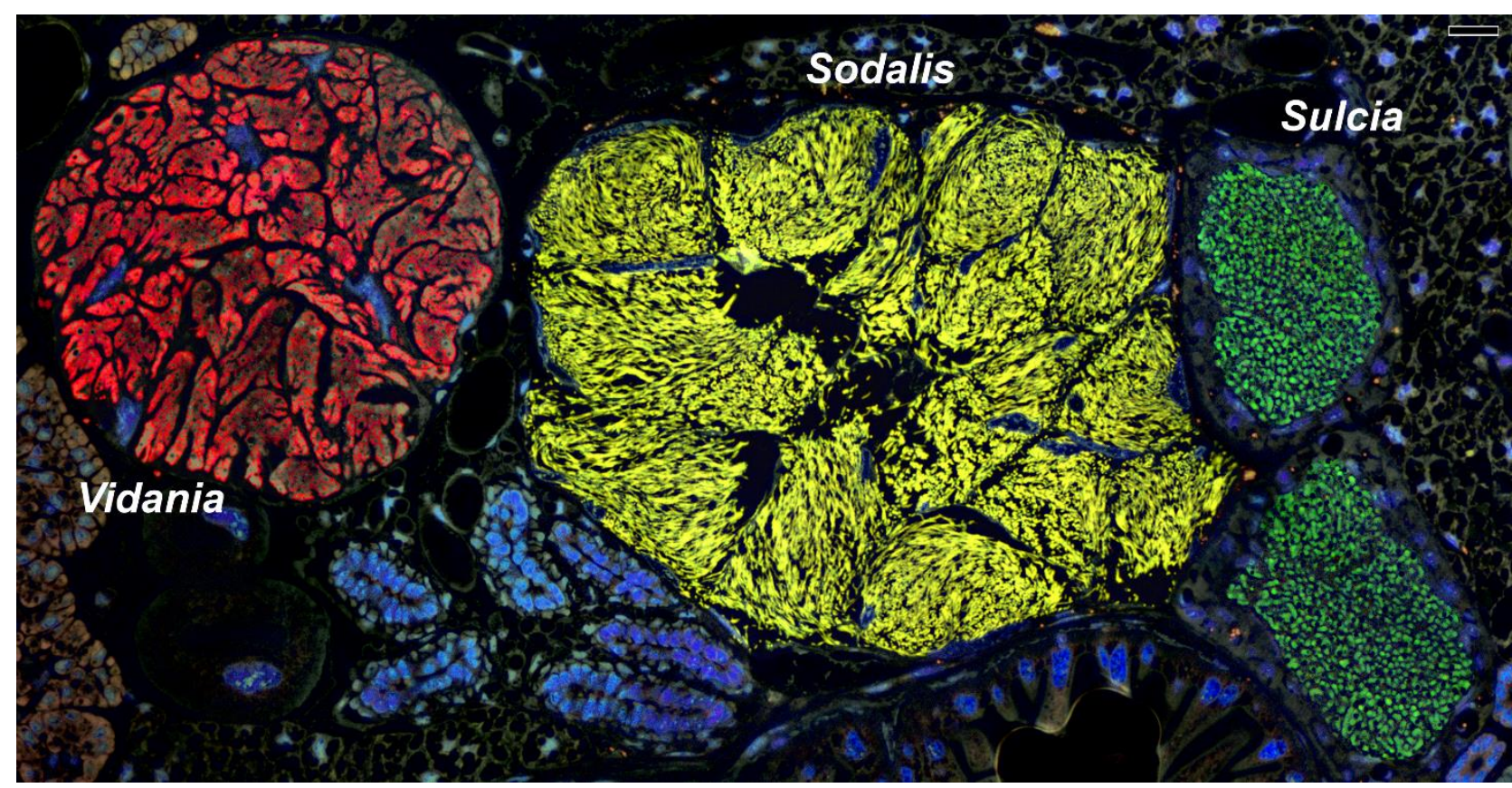

Figure 3. Fluorescent in-situ hybridization (FISH) demonstrates how in C. krueperi, each of its three co-primary symbionts inhabit distinct bacteriomes. Specific probes for Vidania (red), Sulcia (green), and Sodalis (yellow) were used. Blue represents DAPI. Confocal laser microscope (CLM), scale bar $=20 \mu \mathrm{m}$.

located in proximity to each other within the insect abdomens (Figs 3,4). Light microscopy observations have revealed that bacteriomes harboring bacteria Sulcia, Sodalis, and Arsenophonus are made up of several bacteriocytes (Figs 3; 4A), whereas bacteriomes with Vidania are syncytial (Figs 3, 4D). Bacteriomes occupied by Sulcia and Vidania are surrounded by thick (in Sulcia) or thin (in Vidania) monolayered bacteriome sheath (Fig. 4A, C, D, F). In contrast, bacteriomes with Arsenophonus and Sodalis are not covered by epithelial cells (Fig. 3).

Our histological, ultrastructural, and FISH analyses revealed that Sulcia cells are pleomorphic and possess large electron-dense inclusions in their cytoplasm (Figs. 3; 4A-C). Vidania cells are giant and multi-lobed, with numerous electron-dense accumulations in the cytoplasm (Figs. 3; 4DF). The third type of nutritional symbionts - bacteria Sodalis (in C. krueperi, D. pannonica, D. multireticulata, and $R$. edirneus) and Arsenophonus (in D. europaea, R. scytha, and $P$. platypus) are large and elongated (Figs 3, 4J-L). As a rule, their morphology is very similar within species. In particular, morphological data did not suggest the presence of distinct Arsenophonus strains, further supporting our amplicon-based conclusions that different $16 \mathrm{~S}$ OTUs correspond to different rRNA operons of the same strains (Figs 3; 4J-L). The only exception was Sodalis in D. pannonica, where in some individuals, we observed two different morphotypes of these bacteria, differing in size, methylene blue staining intensity, and electron density. These morphotypes share the same bacteriocytes; however, they may be mixed or form aggregates (not shown).
In all species analyzed, bacteriocytes have large, polyploid nuclei, and their cytoplasm is tightly packed with symbionts, ribosomes, and mitochondria (Figs. 3; 4). The density of mitochondria in bacteriocytes with Sulcia and Vidania seemed substantially higher than in bacteriocytes with Sodalis and Arsenophonus. Bacteria Sulcia and Vidania tightly adhere to each other in the cytoplasm of their bacteriocytes, whereas Sodalis and Arsenophonus are somewhat less densely packed (Figs 3; 4A-L). Finally, during ultrastructural observations in C. krueperi and D. europaea, we detected small, rod-shaped bacteria in the cytoplasm of bacteriocytes housed by Sulcia and Vidania symbionts (SI Appendix, Fig. S9). Their shape and size matched Wolbachia, detected in these species using amplicon sequencing.

Besides bacteriomes, Vidania also occupies bacteriocytes in the rectal organ, localized in the invagination of hindgut epithelium (Fig. 4M-O). Both histological and ultrastructural studies made it clear that Vidania cells in the rectal organ differ in shape and size from Vidania localized in the bacteriomes

\section{Dictyopharids developed different modes of symbiont transmission}

Histological observations of serial semithin sections have shown that all nutritional symbionts detected in Dictyopharidae species analyzed are transovarially transmitted between generations. The details of Sulcia, Vidania, and Arsenophonus transmission agree with observations from other Auchenorrhyncha. Strikingly, we observed significant differences in the modes of transmission of Sodalis (Figs 5-8). 


\section{bacteriomes}
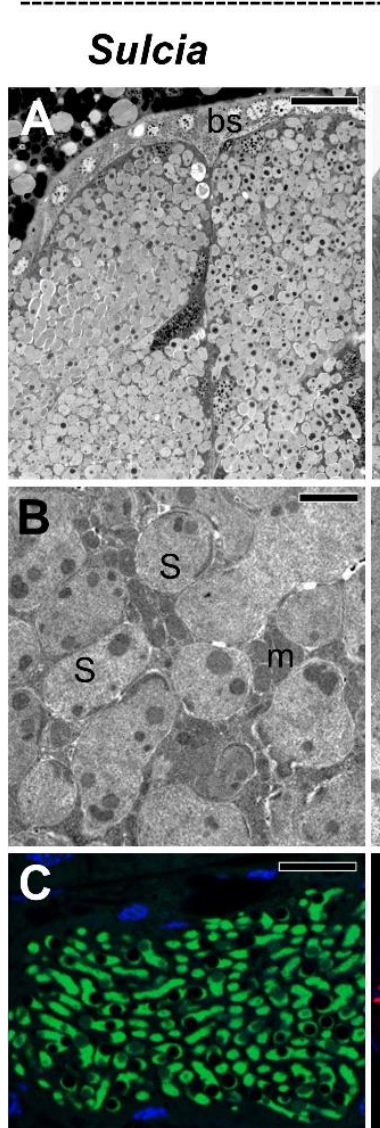

\section{Vidania}
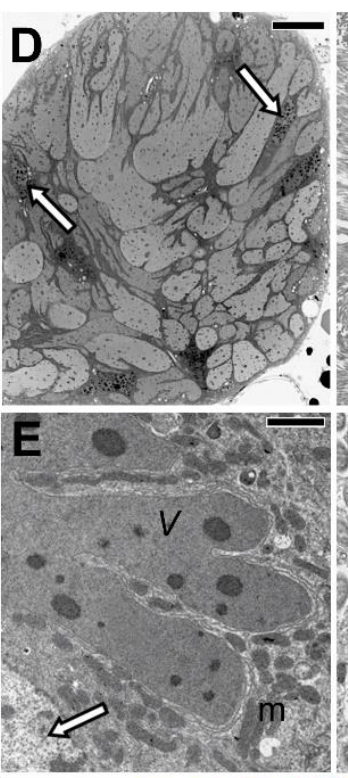

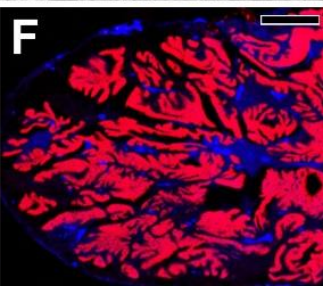

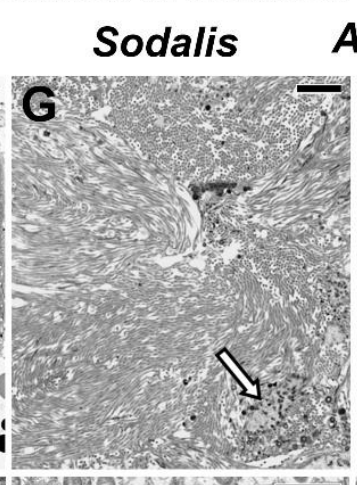

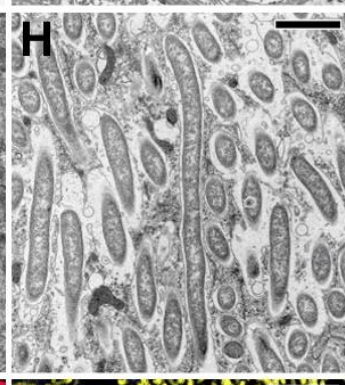

Arsenophonus
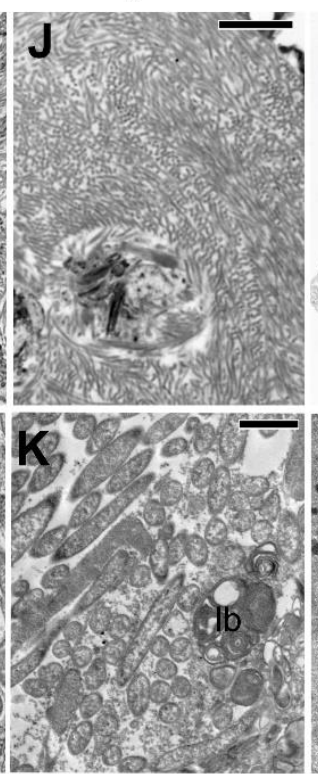
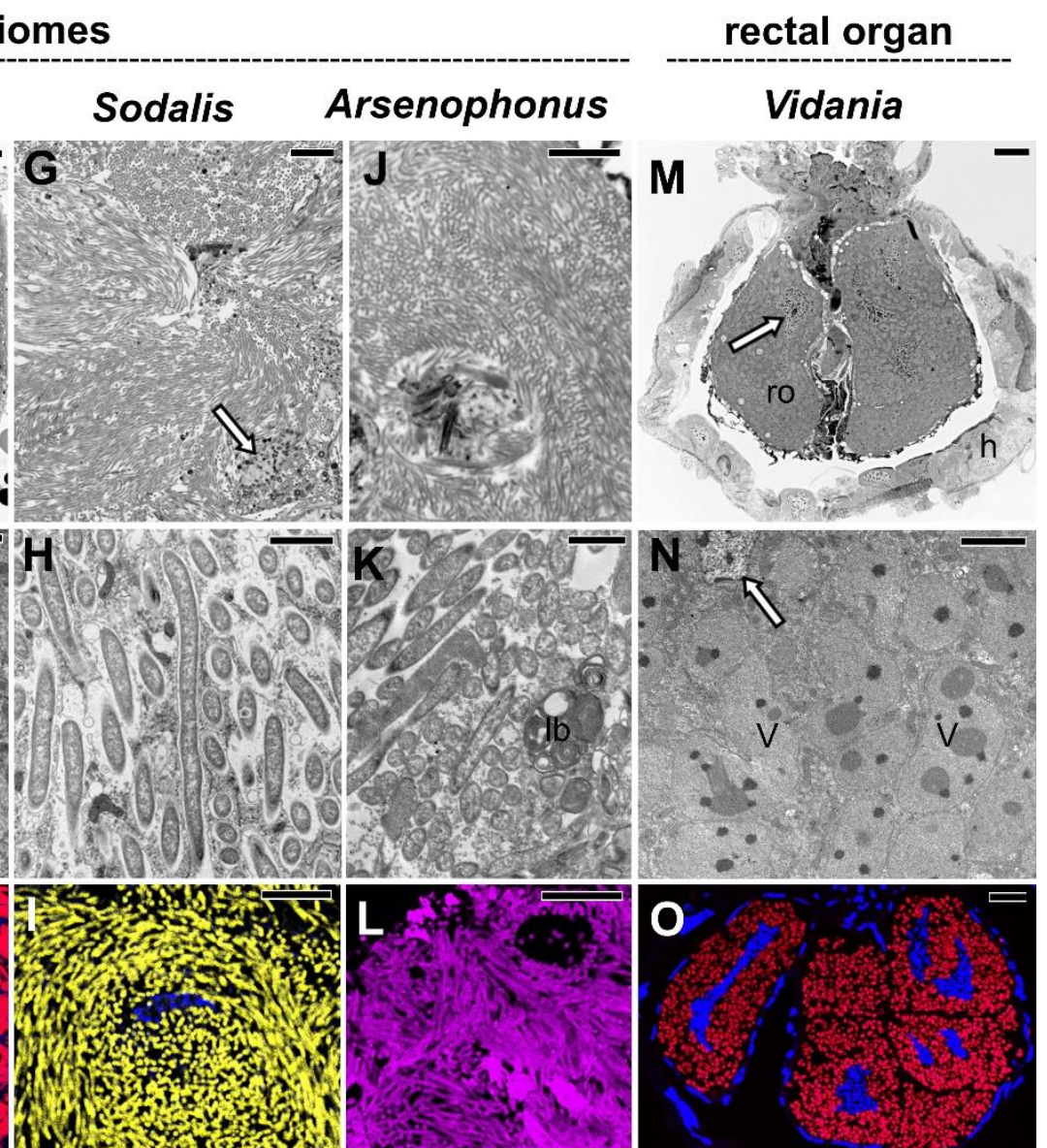

Figure 4. Tissue localization and morphology of symbionts in the Dictyopharidae species examined. Top row (A, D, G, J, M): the organization of symbionts within bacteriomes or the rectal organ. Light microscopy (LM), scale bar $=20 \mu \mathrm{m}$. Middle row (B, E, H, K, N): the ultrastructure of symbiont cells. Transmission electron microscopy (TEM), scale bar $=2 \mu \mathrm{m}$. Bottom row (C, F, I, L, O): fluorescent insitu hybridization (FISH) microphotographs of symbiont cells within the bacteriome or rectal organ. Probes specific to each of the symbionts were used. Blue represents DAPI. Confocal laser microscope (CLM), scale bar $=20 \mu \mathrm{m}$. Insect species: A - D. multireticulata, B, L $-R$. scytha, C, E-I, M, N - C. krueperi, D, O - D. pannonica, J, K - D. europaea. Abbreviations: bs - bacteriome sheath, h - hindgut, lb - lamellar body, $\mathrm{m}$ - mitochondrion, ro - rectal organ, S - Sulcia, V - Vidania, white arrow - bacteriocyte nucleus.

In all species examined, Sulcia and Vidania infect the posterior end of the ovariole, migrating at the same time (Figs 5A; 6A).

In contrast, in C. krueperi, D. pannonica, and D. multireticulata, we observed the separation of symbiont transmission in time and space. Instead of entering the perivitelline space through follicular cells, Sodalis infects the neck region of the ovariole i.e. the part of the ovariole between tropharium and vitellarium. We observed large accumulations of Sodalis cells in ovarioles containing oocytes in the previtellogenic stage of oogenesis. The follicular cells separating the tropharium from the vitellarium are tightly packed with bacteria (Fig 7A-C). This specific region of the ovariole is penetrated by nutritive cords which connect oocytes developing in the vitellarium with trophocytes localized in the tropharium (Fig. 7A-C). Unfortunately, due to the lack of larval stage of species analyzed, we could not observe the migration of Sodalis symbionts from the body cavity to the ovariole. Bacteria Sodalis infect previtellogenic oocytes using nutritive cords
(Fig. 7D-H). They leave the follicular cells en masse, enter the nutritive cord area (Fig. 7D, E) and migrate towards the oocyte along microtubules (Fig. 7F-H). Then, Sodalis cells aggregate in the cytoplasm of the anterior pole of the oocyte (Fig. $7 \mathrm{H}$ ) and stay in this form through the next stages of oogenesis (Fig. 7I). In these species, Sulcia and Vidania symbionts are transmitted to the perivitelline space of choriogenic oocytes (which contain clusters of Sodalis bacteria in the cytoplasm of the anterior pole) by way of the follicular cells at their posterior end (Fig. 8A-C). They closely adhere to each other and form a characteristic "symbiont ball" (Fig. 8D-F).

In contrast to Sulcia, Sodalis, and Arsenophonus, which do not change their shape substantially during migration, Vidania undergoes significant morphological changes. In comparison to lobate Vidania cells within bacteriocytes, migrating Vidania cells (in the cytoplasm of follicular cells and in the "symbiont ball") are smaller and more spherical (Figs 6; 8). Transmitting Vidania cells resemble those occupying the rectal organ. 

made available under aCC-BY 4.0 International license.

A
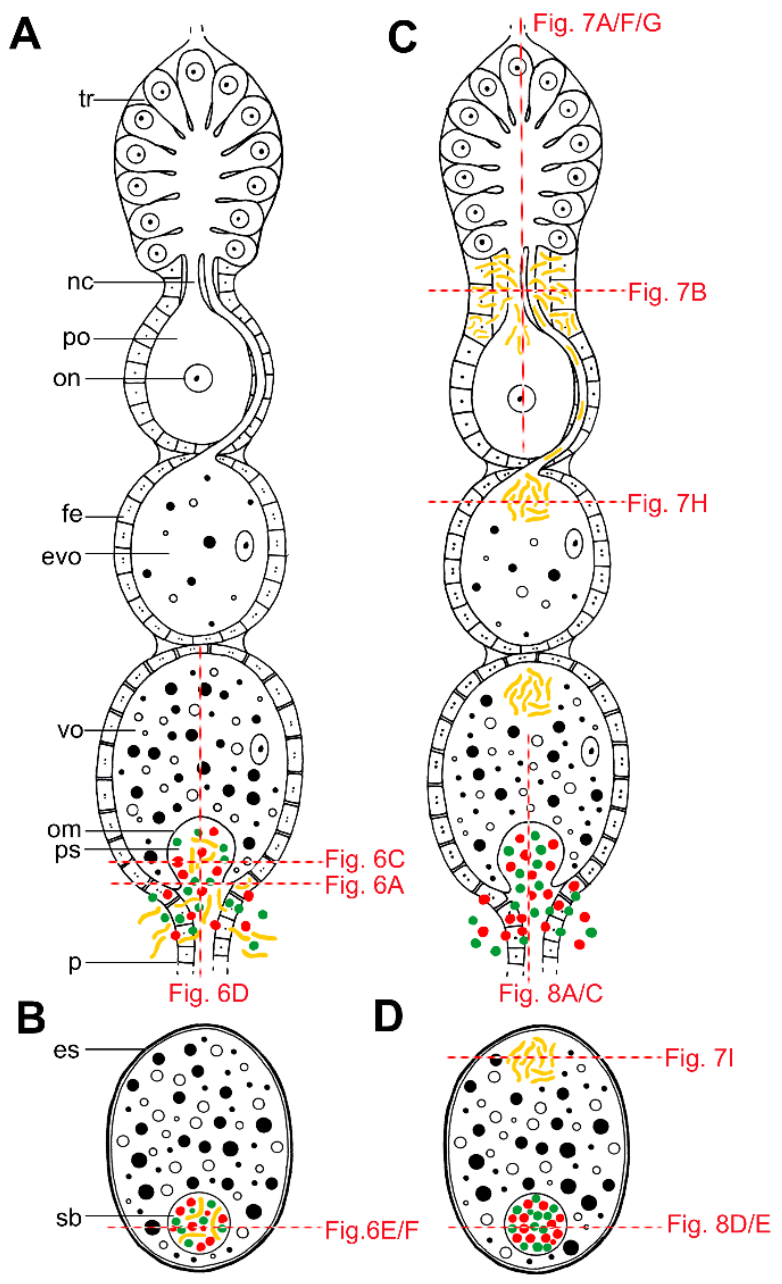

Figure 5. Schematic representation of the alternative modes of the symbiont transmission in Dictyopharidae planthoppers. A. Simultaneous transmission of all types of nutritional symbionts through the follicular epithelium surrounding the posterior pole of terminal oocyte. B. Full-grown oocyte with a symbiont ball containing three types of symbionts. The transmission mode shown in A-B is used by the large majority of Auchenorrhyncha, including subfamily Orgerinae and Arsenophonus-infected D. europaea from subfamily Dictyopharinae. C. Spatially and temporarily separated transmission of different symbionts (Sodalis vs. Sulcia and Vidania). Sodalis infects previtellogenic and early-vitellogenic oocytes, whereas the remaining symbionts invade terminal vitellogenic oocytes. D. Full-grown oocyte with the accumulation of Sodalis bacteria at the anterior pole, and symbiont ball with Sulcia and Vidania cells at the posterior pole. This mode of transmission is unique to Sodalis-infected members of the subfamily Dictyopharinae. Red dashed lines indicate regions shown on panels of Figures 6-8. Abbreviations: $\operatorname{tr}$ - tropharium, nc nutritive cord, po - previtellogenic oocyte, on - oocyte nucleus, fe - follicular epithelium, evo - early-vitellogenic oocyte, vo vitellogenic oocyte, om - oocyte membrane, ps - perivitelline space, es - egg shells, sb - symbiont ball.

\section{Discussion}

Repeated symbiont replacements shape the symbiosis in Dictyopharidae planthoppers

Amplicon sequencing, metagenomics, and microscopy data agreed that all studied species of Dictyopharidae host heritable nutritional symbionts Sulcia and Vidania. We know that Sulcia infected the common ancestor of all Auchenorrhyncha that lived about 300 MYA, and Vidania, together with spittlebug-associated Zinderia and leafhopper-associated Nasuia, appears to represent a similarly ancient lineage $(2,23,24)$. Both of these symbionts are retained in many modern planthoppers (15, 20-22), but so far, their genomes have been characterized in only one other planthopper species, O. filicicola OLIH (Cixiidae). We found surprisingly few differences in the symbiont genome organization or contents between Dictyopharidae and OLIH, despite them being separated by about $200 \mathrm{MY}$ of evolution (2). While in Dictyopharidae, symbiont genomes are somewhat smaller than those in OLIH (142-148 kb vs $156 \mathrm{~kb}$ for Sulcia, 122-126 kb vs $136 \mathrm{~kb}$ for Vidania), they are colinear and encode essentially the same set of nutrient biosynthesis genes. Sulcia-CALKRU has the most compact genome out of many strains of this symbiont characterized so far. Vidania falls among bacteria with the smallest known genomes, alongside other hemipteran symbionts: Nasuia $(\geq$ $110 \mathrm{~kb})$, Tremblaya ( $\geq 138 \mathrm{~kb})$, Carsonella $(\geq 164 \mathrm{~kb})$, and Hodgkinia $(\geq 144 \mathrm{~kb}$, but note that individual genomes in fragmented complexes can be much smaller) (7, 25-28).

The reduction of symbiont genomes in long-lasting symbiotic interactions is a natural consequence of the lack of DNA repair genes or recombination, allowing for the progressive accumulation of deleterious mutations. Combined with the increase in rates of evolution, this leads to rapid loss of genes and pathways and is thought to negatively affect the function and efficiency of others, including those involved in essential nutrient biosynthesis (29-31). This opens up a path to their complementation or replacement by newly arriving microorganisms, more versatile and efficient. From the evolutionary perspective, both the complementation and replacement of "old" symbionts by "new" ones should allow host insects to refresh and reset their biosynthetic capacity and likely positively influence fitness and perhaps extend ecological niche $(7,23)$. The radiation of insect clades whose ancestors acquired new symbionts, including cicadas, sharpshooter leafhoppers, aphids, carpenter ants, and others, is suggestive of a competitive edge that the nutritional symbiont acquisition or replacement can provide. 
bioRxiv preprint doi: https://doi.org/10.1101/2021.04.07.438848; this version posted April 9, 2021. The copyright holder for this preprint (which was not certified by peer review) is the author/funder, who has granted bioRxiv a license to display the preprint in perpetuity. It is made available under aCC-BY 4.0 International license.

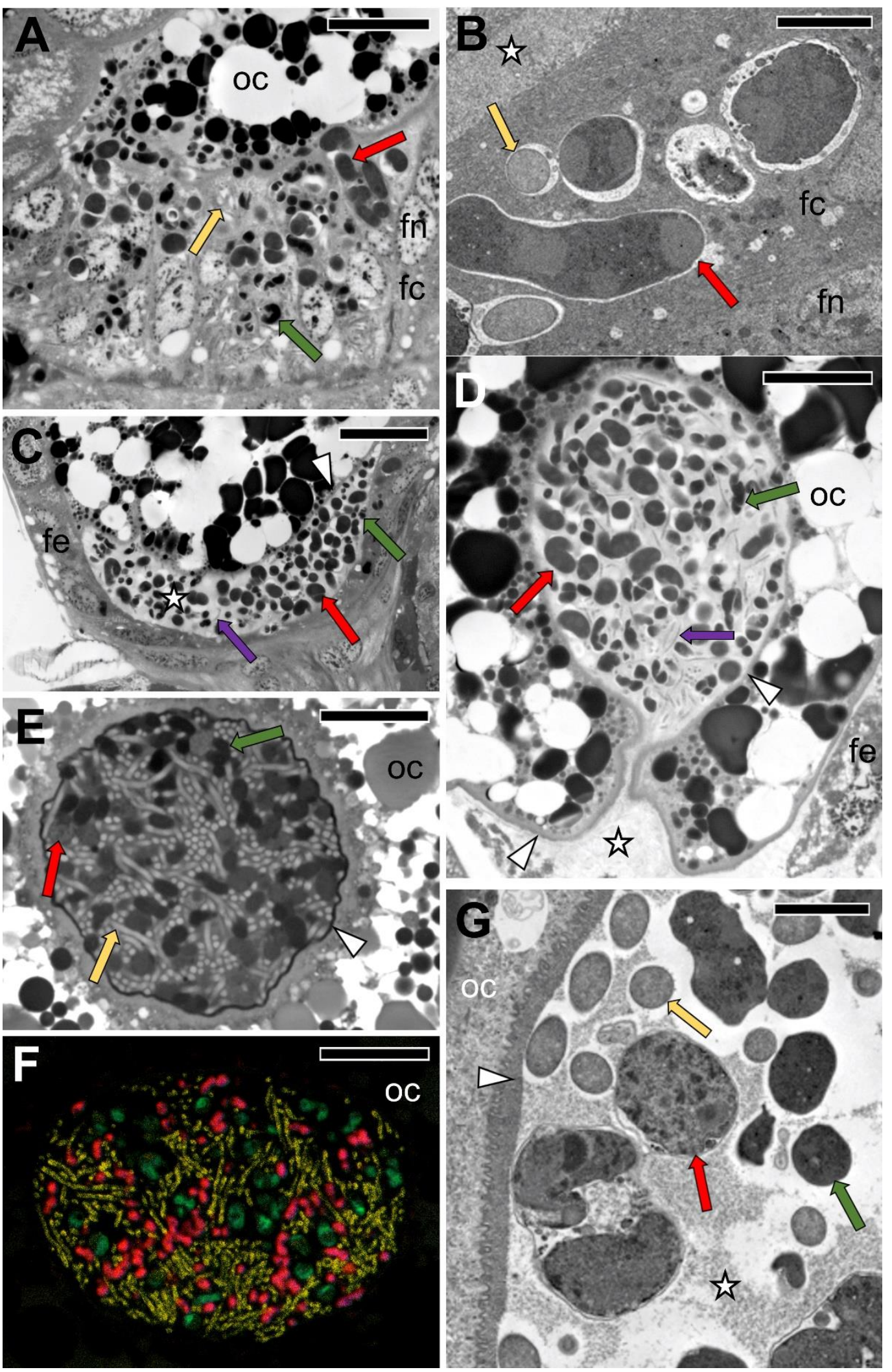

Figure 6. The simultaneous transmission of three symbionts in selected Dictyopharidae, as shown in Fig. 5A-B. A. The migration of Sulcia, Vidania, and Arsenophonus through follicular cells surrounding the posterior pole of the terminal oocyte. D. europaea, light microscopy $(\mathrm{LM})$, scale bar $=20 \mu \mathrm{m}$. B. Sodalis and Vidania in the cytoplasm of the follicular cell. R. edirneus, TEM, scale bar $=2 \mu \mathrm{m}$. C, D. Symbiotic bacteria in the perivitelline space. D. europaea, LM, scale bar $=20 \mu \mathrm{m}$. E. A 'symbiont ball' containing bacteria Sulcia, Vidania, and Sodalis in the deep depression of the oolemma at the posterior pole of the terminal oocyte. R. edirneus, LM, scale bar $=20 \mu \mathrm{m}$. F. In situ identification of symbionts in the "symbiont ball" within the terminal oocyte. $R$. edirneus, CLM, scale bar $=20 \mu \mathrm{m}$. G. Fragment of the "symbiont ball" in the perivitelline space. $R$, edirneus, TEM, scale bar $=2 \mu \mathrm{m}$. Abbreviations: fc - follicular cell, fn - the nucleus of the follicular cell, oc - oocyte, asterisk - perivitelline space, arrowhead - oocyte membrane, red cells/arrows - Vidania, green cells/arrows Sulcia, yellow cells/arrows - Sodalis, purple arrows - Arsenophonus 

made available under aCC-BY 4.0 International license.

We found that in addition to the two ancestral symbionts, all studied Dictyopharidae species harbor heritable, bacteriocyte-associated enterobacteria, either Sodalis or Arsenophonus, apparently acquired several times independently. These two microbial clades have repeatedly infected diverse insects including multiple hemipteran lineages. Sodalis is known as a nutritional symbiont of blood feeding flies and lice, lygaeid bugs, and weevils (32-34), but a strain has also been isolated from a human wound (35). Arsenophonus has assumed different functions in different hosts: some strains are obligatory, heritable nutritional mutualists of blood sucking flies or other insects, others facultative symbionts, reproductive manipulators, or insectvectored plant pathogens (2, 36-39). In Auchenorrhyncha, Sodalis and Arsenophonus usually associate with ancestral symbionts, presumably complementing them (34). For example, they have been reported alongside ancestral symbionts in leafhoppers Macrosteles laevis and Cicadella viridis and in the spittlebug Aphrophora quadrinotata (10, $12,40)$. Other times, they appear to have replaced one of the ancient symbionts. For example, Koga and colleagues (12) reported a link between the loss of Zinderia and the acquisition of Sodalis in modern Philaenini spittlebugs. There is little information on how often such replacements happen; but we think that serial replacements are the most likely explanation for the diversity of Sodalis/Arsenophonus symbionts within the studied Dictyopharidae. Likewise, Husnik and McCutcheon (26) suggested that this is a plausible explanation for the diversity of enterobacterial symbionts within mealybugs.

Unfortunately, 16S rRNA data provide limited phylogenetic resolution, and the scarcity of genomic references for these broadly distributed and significant symbionts makes it hard to resolve relationships among strains conclusively. However, the distribution of symbiont strains on the host phylogeny, alongside differences in how Sodalis in the two subfamilies is transmitted, makes it apparent that the current enterobacterial symbiont distribution across the studied planthopper species is the result of not less than four infection/replacement events.
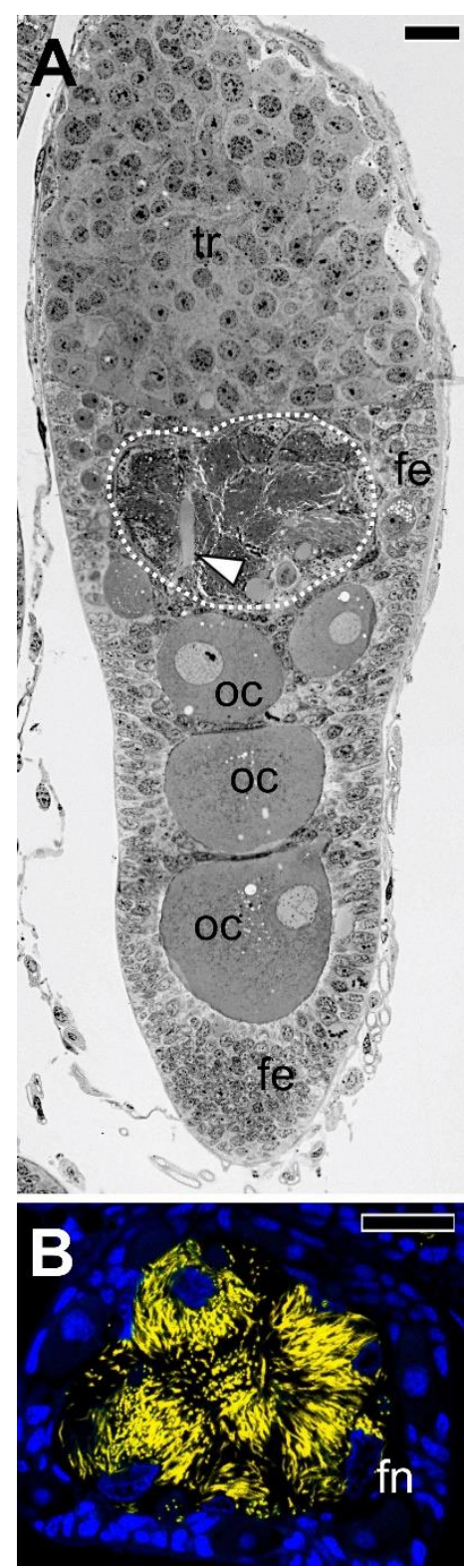
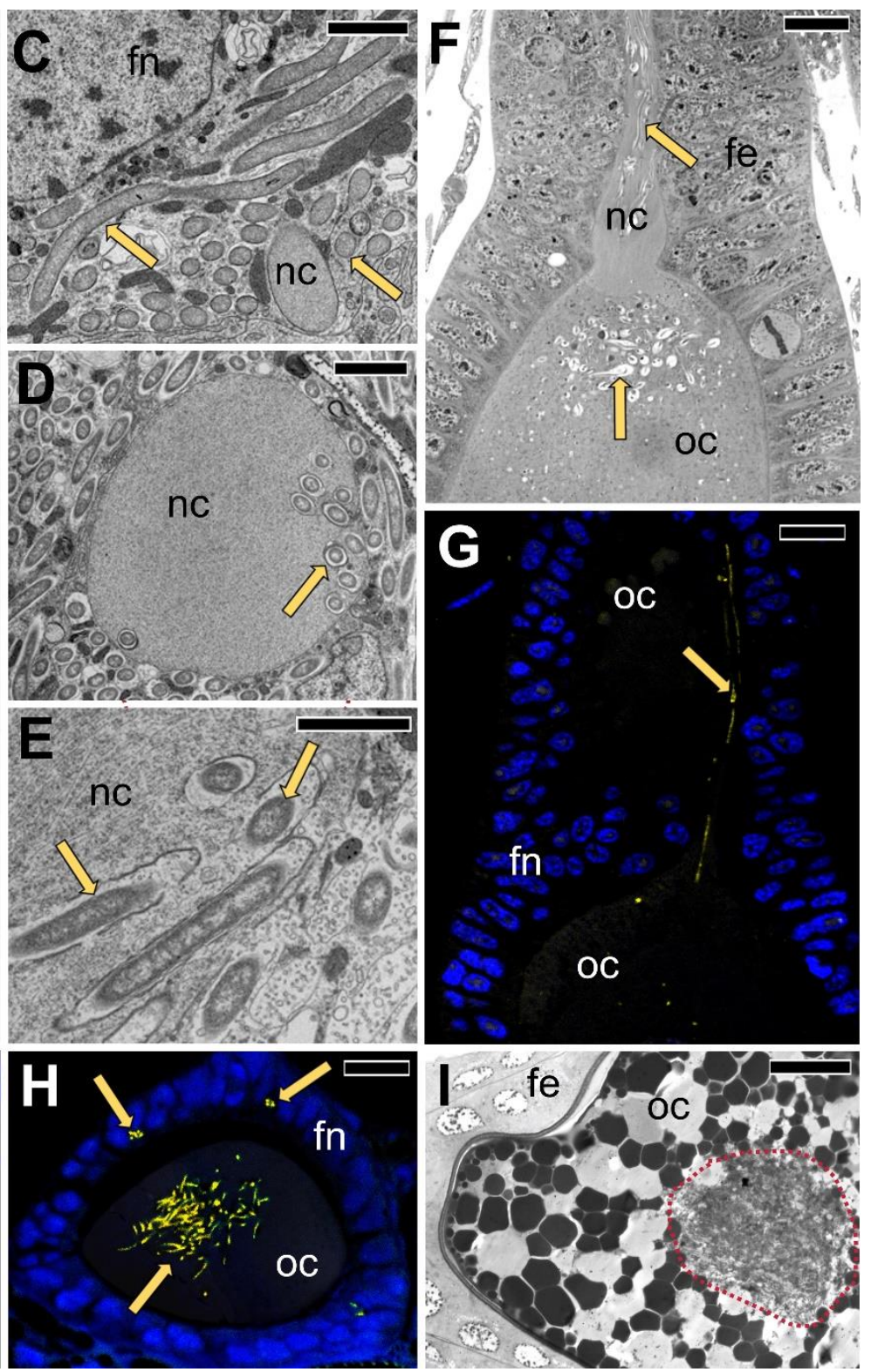
Figure 7. Transovarial transmission of Sodalis into the anterior pole of the developing oocyte in representatives of Dictyopharinae subfamily. A. Longitudinal section through the ovariole in the previtellogenesis stage. Note numerous Sodalis cells in the neck region of the ovariole (area surrounded with white dotted line). D. pannonica, LM, scale bar $=20 \mu \mathrm{m}$. B. Cross-section through the neck region of the ovariole filled with prefollicular cells with bacteria Sodalis (marked in yellow), C. krueperi, confocal microscope, scale bar $=20 \mu \mathrm{m}$. C. Fragment of the prefollicular cell with Sodalis occupying the neck region of the ovariole. D. multireticulata, TEM, scale bar $=2 \mu \mathrm{m}$. D. Cross-section through the nutritive cord surrounded by Sodalis. Note Sodalis cells in the cytoplasm of the nutritive cord. C. krueperi, TEM, scale $b a r=2 \mu \mathrm{m}$. E. The higher magnification of Sodalis bacteria migrating via nutritive cord to the oocyte. $C$. krueperi. TEM, scale bar $=2$ $\mu \mathrm{m}$. F, G. The transport of Sodalis via nutritive cord to the previtellogenic oocyte. C. krueperi. F. LM, scale bar $=20 \mu \mathrm{m}$. G. CLM, scale bar $=20 \mu \mathrm{m}$. H. Accumulation of Sodalis cells in the cytoplasm of the anterior region of the previtellogenic oocyte. C. krueperi, CLM, scale bar $=20 \mu \mathrm{m}$. I. Accumulation of Sodalis in the cytoplasm of the choriogenic oocyte. D. multireticulata, LM, scale bar $=20 \mu \mathrm{m}$. Abbreviations: $\mathrm{fe}$ - follicular epithelium, fn - the nucleus of a follicular cell, nc or arrowhead - nutritive cord, oc - oocyte, yellow cells/arrows - Sodalis.

The diversity of gammaproteobacterial symbionts, including also Serratia and Symbiopectobacterium in mealybugs, aphids, and other systems, raises questions about the actual dynamic, temporal scales of infections and replacements with these microbes. They can only be addressed using systematic genomics-enabled surveys within insect clades.

\section{Remarkable conservation of tissue localization and functions in dynamic planthopper symbioses}

In all Dictyopharidae species analyzed, as in other planthoppers examined previously, the ancestral symbionts Sulcia and Vidania are localized in distinct, spatially separated bacteriomes within the insect body cavity $(15,41-$ 43). This contrasts with the situation in Cicadomorpha, where the ancestral symbionts always inhabit the same bacteriome: Sulcia, as a rule, occupies bacteriocytes within the outer layer of the bacteriome, whereas the co-residing ancestral symbiont (Nasuia, Zinderia or Hodgkinia) occupies the central portion $(11,27,44)$. The differences in the spatial organization of symbiont-housing organs may be due to chance, but could also be related to their mutual relations and nutritional capabilities. In Cicadomorpha, co-primary symbionts are thought to share some cofactors and metabolites, mainly involved in the biosynthesis of energetically expensive amino acids such as methionine or histidine (4), and this could be facilitated by the proximity of bacteriocytes inhabited by different symbionts.

While the ancestral hemipteran symbionts are usually housed in dedicated insect cells - bacteriocytes, the localization of more recently acquired symbionts may differ. Gammaproteobacterial symbionts, including Arsenophonus and Sodalis, localize differently in different insect hosts. The gammaproteobacterial symbionts of Dictyopharidae, despite their varied origins, are always located within bacteriomes separated from, but adjacent to, those occupied by Sulcia and Vidania. In other Auchenorrhyncha, they can also occur in separate bacteriomes, or alternatively, colonize distinct bacteriocytes within existing bacteriomes, but they are also observed in the cytoplasm of the same bacteriocytes as ancestral symbionts, or even inside other symbionts' cells $(10,11,19)$. In other insects, they may be dispersed across host tissues other than bacteriocytes, including gut epithelium cells, fat body cells, and hemolymph $(45,46)$. Interestingly, in some systems, independently acquired microbes tend to inhabit the same tissue compartments, suggestive of pre-adaptations that make it a particularly hospitable place: gammaproteobacterial symbionts of Pseudococcinae mealybugs, always residing inside the cells of the ancient Tremblaya symbiont, are a striking example (26).

In females of all Dictyopharidae, similarly to previously studied planthopper species, we observed two morphotypes of Vidania occupying distinct bacteriomes in the body cavity and rectal organ. Buchner (15) suggested that symbionts derived from the rectal organ are the infectious form of the same giant lobed symbionts that occur in the bacteriome. Similarly, Bressan and Mulligan (42) hypothesized the functional specialization of Vidania bacteriomes into organs containing non-infectious symbiont cells with prominent metabolic functions, and the rectal organs with Vidania cells serve for the transmission to the progeny. Future transcriptomic studies should clarify the roles of these morphotypes, but, our microscopic observations agree that Vidania cells which are transmitted to the ovary are of rectal organ origin.

In Cicadomorpha, Sulcia encodes genes involved in the biosynthesis of seven to eight amino acids, while the companion symbiont is responsible for the provisioning of the remaining two or three (histidine, methionine and sometimes tryptophan). In Fulgoromorpha, the relative contributions of the companion symbionts are reversed: the ability to synthesize arginine, lysine, phenylalanine, and threonine has been lost by Sulcia and they are produced by Vidania instead. Notably, the near-identity of biosynthetic capabilities in Dictyopharidae and the divergent cixiid, $O$. filicicola, reveals how stable their ancestral nutritional endosymbionts can be over $200 \mathrm{MY}$ of evolution alongside changing gammaproteobacterial partners. The consistent differences in Sulcia biosynthetic capabilities between Cicadomorpha and Fulgoromorpha suggest that the two clades have separated soon after the acquisition of the ancestral beta-symbiont - assuming that there was indeed one, as proposed but not unambiguously demonstrated (23). 

made available under aCC-BY 4.0 International license.

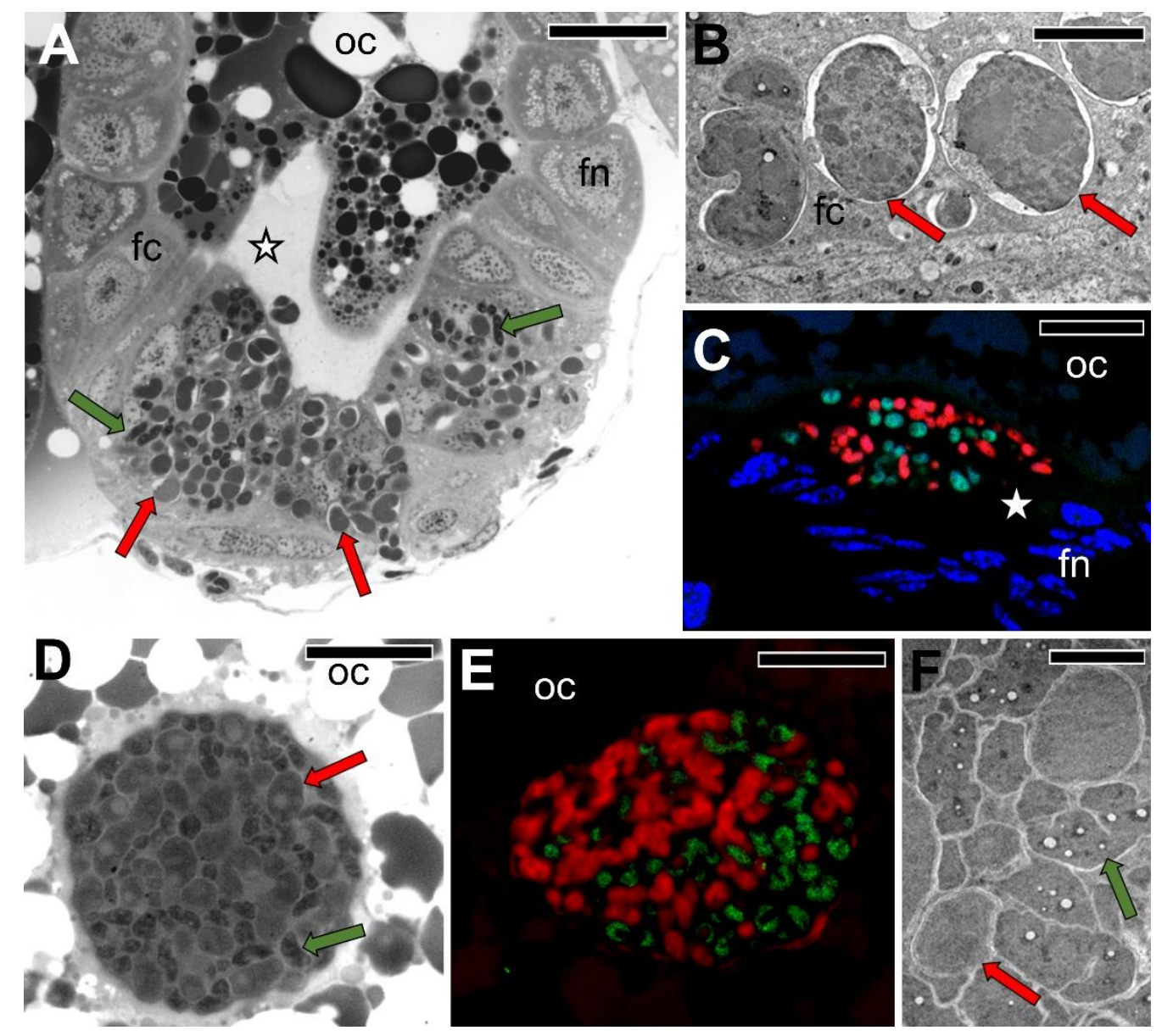

Figure 8. Transovarial transmission of Sulcia and Vidania into the posterior pole of the terminal oocyte in representatives of Dictyopharinae subfamily. A. The migration of Sulcia and Vidania to the perivitelline space through the follicular epithelium surrounding the posterior pole of the terminal oocyte. D. multireticulata, LM, scale bar $=20 \mu \mathrm{m}$. B. Vidania in the cytoplasm of the follicular cell. $D$. pannonica, TEM, scale bar $=2 \mu \mathrm{m}$. C. Accumulation of Sulcia (green) and Vidania (red) in the perivitelline space. C. krueperi. Confocal microscope, scale bar $=20 \mu \mathrm{m}$. D. A "symbiont ball" containing Sulcia and Vidania in the deep depression of the oolemma at the posterior pole of the terminal oocyte. D. multireticulata, LM, scale bar $=20 \mu \mathrm{m}$. E. In situ identification of symbionts in the "symbiont ball" in the mature oocyte of $C$. krueperi. CLM, scale bar $=20 \mu \mathrm{m}$. F. Fragment of "symbiont ball". D. multireticulata. TEM, scale bar $=2 \mu \mathrm{m}$. fc follicular cell, fn - the nucleus of a follicular cell, oc - oocyte, asterisk - perivitelline space, red cells/arrows - Vidania, green cells/arrows Sulcia.

Later, stochastic or other factors must have caused differential gene and pathway loss among the partner symbionts in the ancestors of the two infraorders.

In planthoppers, at least those studied to date, Bvitamin biosynthesis has been outsourced to additional, gammaproteobacterial symbionts, for example Purcelliella in O. filicicola or Arsenophonus in Nilaparvata lugens (47, 48). Indeed, all characterized planthopper-associated Sodalis and Arsenophonus strains possess largely complete sets of genes involved in riboflavin and biotin production. Additionally, Sodalis of C. krueperi and Arsenophonus of $R$. scytha may be able to synthesize pyridoxine and folate, respectively. A similar biosynthetic potential was found in Sodalis-related symbionts of mealybugs (26) and Arsenophonus living in symbiotic relations with other insects such as the wasp Nasonia vitripennis, and many whiteflies and louse flies $(33,34,49)$. Additionally, both Sodalis and Arsenophonus possess some genes from essential amino acid biosynthesis pathways. Most of them overlap with those retained on the Vidania genome. However, in C. krueperi, Sodalis might complement Vidania in the biosynthesis of methionine and in D. multireticulata, Sodalis retains two genes necessary in lysine production that underwent pseudogenization in the Vidania genome, suggesting that different microbes may complement each other in the nutrient biosynthesis. Such biosynthetic pathway complementarity between the host and symbionts has been demonstrated experimentally in the mealybug system (50) but is likely to be a more universal phenomenon. It would be interesting to explore more broadly whether there is a general trend towards complementarity, how it is affected by serial co-primary symbiont replacements, and whether it can facilitate the replacement of ancient symbionts. 

made available under aCC-BY 4.0 International license.

\section{Newly arriving symbionts follow different strategies of transmission across host generations}

The crucial role of nutritional symbionts in hostinsect biology is evidenced by their vertical transmission, which ensures the presence of symbionts in subsequent generations. Insects have adopted multiple vertical transmission strategies $(16,17)$, but the transovarial transmission is probably the most reliable means of providing the full symbiont complement to all offspring. In Auchenorrhyncha, ancient symbionts are transmitted in a consistent, conserved way. In all species studied so far, they cross the follicular cells surrounding the posterior pole of the vitellogenic oocytes and then form the "symbiont ball" near its posterior end (17). Additional symbionts generally follow the same path $(11,44,51)$; this includes Arsenophonus and Sodalis in some of the Dictyopharidae presented here. The discovery that in other Dictyopharidae species, Sodalis have adopted a very different transmission strategy, invading the "neck region" of the ovariole from where it is carried to the cytoplasm of previtellogenic oocytes via the nutritive cords, expands our understanding of the diversity of strategies that newly arriving insect symbionts may adopt. The fact that closely related microbes adopted different strategies in related host species is particularly notable. Nonetheless, the spatiotemporal separation of transovarial symbiont transmission is not unique to this insect clade. For example, in the planthopper Cixius nervosus, one of its symbionts, unidentified so far, was observed to infect undifferentiated cystocytes, while others full-grown oocytes (17). At the same time, Müller (22), who originally reported different infection strategies in Dictyopharidae, and Buchner (15), who in his pivotal book compared these data against vast amounts of other observations, emphasized their uniqueness.

In other groups of hemipterans and more divergent insects, gammaproteobacterial nutritional symbionts have adopted a wider range of strategies. For instance, in some scale insects, bacteria invade larval ovaries and germ cells at a very early stage, before they differentiate into oocytes and trophocytes, and are later present in all germ cells in the ovariole (17). In some heteropteran bugs but also carpenter ants, symbiotic gammaproteobacteria directly infect early previtellogenic oocytes: infect follicular cells, gather in their cytoplasms, and then enter the oocyte's cytoplasm via an endocytic pathway. In both cases, initially, symbionts are dispersed in the entire ooplasm, but during vitellogenesis they accumulate and form "symbiont ball" $(52,53)$. In some host lineages, enterobacterial symbionts have adopted more exotic transmission modes. In Cicadella viridis and Macrosteles laevis, gammaproteobacterial symbionts (Sodalis and Arsenophonus, respectively) live inside and are moved to the oocyte within Sulcia cells. In the scale insect Puto superbus, whole, intact bacteriocytes containing Sodalis are transferred into oocytes $(10,40,54)$. In turn, in tsetse flies, Sodalis glossinidius is vertically carried into developing larva via milk gland secretions (55).

Fitness differences between these alternative modes of transmission are not apparent. Theoretically, the early presence of symbionts in oocytes could contribute to their faster development, but there is no evidence supporting this hypothesis. It is also likely that the transmission modes vary in efficiency, including in the proportion of cells departing from the bacteriomes that arrive within the oocytes, or the energy needed for each symbiont cell to complete the journey. It is also probable that there are differences in the maintenance costs of the mechanisms necessary to move the cells between bacteriomes and oocytes, and later, to the target regions of the developing embryo. Transporting gammaproteobacterial symbionts along the same path as Sulcia and Vidania could theoretically allow the host to reuse the existing cellular machinery in a relatively efficient manner, but that would depend on the specificity of the mechanisms, something that we have virtually no knowledge of. Yet another aspect relevant to fitness, especially in the long run, is the symbiont bottleneck size, which may affect the strength of genetic drift and the symbiont evolutionary rates (51). However, at the moment, we know little about the magnitude of any such differences, or how important they might be. But ultimately, and most critically, all mechanisms appear to result in reliable transmission of all symbionts to every offspring.

Why do some symbionts adapt different ways of transmission then others, then? We suspect that this is not typically the outcome of selection, but rather a combination of pre-adaptations, existing constraints, and chance events. The transmission strategy, but also final tissue localization, could be related to the microbe's biology prior to the transition to the endosymbiotic lifestyle. The presence of specific genes or pathways in the microbe's genome, and mechanisms that direct them towards, or facilitate entry into, certain cell types, are likely to drive the differences (56). At the same time, the re-utilization of existing host-encoded mechanisms, including those for the transmission of older symbionts, is likely to play a significant role, probably increasing over time as symbiont genomes degenerate and erode away. For example, Spiroplasma and Wolbachia were shown to utilize the receptors involved in the transport of vitellogenin (protein precursors of yolk) into oocytes to enable entry into developing eggs $(57,58)$. Unfortunately, we can only speculate about the evolutionary processes that have led to this. We suspect that stochastic processes - random changes affecting the affinity between host and symbiont structural molecules at early stages of infection - could play a significant role in determining the way the symbionts end up transmitting. 


\section{How to succeed as a newly arriving heritable symbiont}

Transmission between the host generations is a critical challenge for a heritable nutritional endosymbiont, but only one of several facing the microbe transitioning towards obligate symbiosis (59). Yet, despite the multitude of challenges, it appears that successful infections and replacements are relatively common, probably more so than thought until recently $(13,26,60)$. Then, what is the recipe for the successful colonization of the host by the new symbiont - one leading to a stable, long-term nutritional endosymbiotic association?

To begin, it seems advantageous for the colonizing microbe to be a pathogen - a product of selection for the ability to gain entry into hosts, overcome or avoid barriers (cuticle) and defenses (behaviour, immune system), and access host resources. Infections would often lead to either disease and death of the host, or alternatively, the clearing of the pathogen by the host immune system. However, lowvirulence chronic infections, like those established in some insects by Sodalis praecaptivus (61), could provide ample opportunities for gaining entry into oocytes using some existing mechanisms - the first step of transition into a heritable associate. Pathogenic ancestry has been demonstrated for multiple fungal symbionts of cicadas, all hauling from specialized cicada-pathogenic lineages of the genus Ophiocordyceps (13), and suspected for bacterial genera that established symbioses repeatedly, including Sodalis, Arsenophonus, or Symbiopectobacterium (37, 60). Although our knowledge of natural strain-level variation is currently limited and this prevents the reconstruction of evolutionary patterns, many symbionts are thought to derive from versatile opportunists similar to $S$. praecaptivus, isolated from a human wound resulting from impalement on a tree branch and thus apparently capable of thriving in very different environments, and falling near the base of the Sodalis-allied symbiont phylogeny $(35,62)$.

Once inside the host, the aspiring nutritional endosymbiont needs to establish residence within specific host organs or tissues and adopt a reliable way of transmitting across host generations. This could be a gradual process with various intermediate stages: for example, the microbe might initially colonize fat body cells that would only later evolve into bacteriocytes (63). As discussed previously, even when related organisms interact, different strategies can be successful. The alternative transmission patterns of Sodalisallied symbiont of Dictyopharidae are not the only example. For instance, in cicadas, following Hodgkinia replacement, Ophiocordyceps symbionts may reside either in the external layer of the same bacteriomes as the ancient bacterial symbionts, or in a new type of bacteriome (13).

Another aspect important for the long-term success of a recent infection is its effect on the host fitness $(64,65)$. For the infection to be stable, the symbiont maintenance costs should be counteracted by its positive effects on the host biology. Although stochastic effects such as genetic drift can, in principle, lead to the spread and fixation of a deleterious change (51), the odds of success are clearly greater for the symbiont that positively influences host fitness. The broad metabolic repertoire and efficiency of newly arriving microbes, including their ability to produce essential amino acids and vitamins $(13,34)$, may constitute obvious benefits to the hosts that lack these nutrients, or are limited by the supply from inefficient, degraded, older symbionts. In some cases, other symbiont-conferred benefits, including the production of defensive compounds (66) may also play a role. Through such effects, the newly arriving symbiont may either complement or replace older symbionts.

Once the symbiont establishes, an unavoidable fate appears to be the degeneration of its genome: rapid pseudogenization and loss of genes and pathways leading to genome shrinkage and overall decrease in function. Some symbionts, for example, Sulcia and Vidania of dictyopharids, may arrive at the stage where the loss of the remaining small set of critically important genes is essentially halted over tens or hundreds millions of years, but that appears quite uncommon. As they degenerate, symbionts are increasingly dependent on host-encoded mechanisms and products and may ultimately reach a comparable level of dependence as cellular organelles $(30,31)$. These mechanisms could potentially be reused by different symbionts that co-infect or newly colonize the host. On the other hand, it was shown that two ancient symbionts in a leafhopper rely on different support mechanisms (67). Likewise, the Sodalis-allied Baumannia symbiont that replaced one of them (Nasuia) in a lineage of sharpshooter leafhoppers is supported by a very different set of host genes, suggesting de novo evolution and adaptation of the host support mechanisms (18). However, it is plausible that during serial replacements by related bacteria, which seems to be taking place in dictyopharids or mealybugs, it is easier to repurpose the existing symbiont support mechanisms. Once again, we suspect that both the timing and order of gene loss and the recruitment of specific host support mechanisms may be a largely stochastic process, with chance events such as random mutations and genetic drift determining the outcomes. The microbe's versatility in utilizing host mechanisms may increase its odds of establishing and surviving for a long time as a key part of a successful two- or multi-partite symbiosis. But regardless, once the symbiont establishes within the host, it seems unable to escape the path of genomic degradation and increasing dependence which (23) graphically described as "the rabbit hole".

The list of microbes that are known to have survived as endosymbionts for an evolutionarily significant amount of time is not very long, and does include the ancestral symbionts of various sap-feeder and blood-feeding insect clades. A large proportion of them, including Buchnera, Baumannia, Blochmannia, and Wigglesworthia, have been 
recently identified as members of the Sodalis clade (62). We do not understand what has allowed these symbionts to reach the stable stage (68) rather than degenerate away. But between the high mutational pressure, increasing with progressing genome degeneration, and the constant exposure to new pathogenic and opportunistic microbes, it is becoming increasingly apparent that the majority of gammaproteobacterial symbioses are relatively short-lived. Except for the selected few, the vast majority of such symbionts will succumb to the cycle of serial symbiont replacements.

\section{Material and Methods}

\section{Study species}

We investigated symbioses in seven planthopper species from two subfamilies within the family Dictyopharidae. From subfamily Dictyopharine, we characterized four species: Callodictya krueperi (Fieber, 1872), Dictyophara pannonica (Germar, 1830), Dictyophara multireticulata Mulsant \& Rey, 1855, Dictyophara europaea (Linnaeus, 1767). From Orgeriinae, we studied three species: Ranissus (Schizorgerius) scytha (Oshanin, 1913), Ranissus (Ranissus) edirneus (Dlabola, 1957) and Parorgerius platypus (Fieber, 1866). Adult specimens were sampled from a single population of each species in either Bulgaria or Poland between 2016 and 2018 (Dataset Table S1), preserved in $80 \%$ ethanol or glutaraldehyde, and stored at $4{ }^{\circ} \mathrm{C}$ until processing. Representative specimens from each species were identified based on morphological characteristics, and identities confirmed using marker gene sequencing.

\section{Amplicon-based microbiome screen}

Library preparation and sequencing. To obtain the preliminary picture of the microbiomes in the seven experimental species, we sequenced amplicons for the V4 hypervariable region of the bacterial 16S rRNA gene, simultaneously confirming the specimen identity by sequencing amplicons for partial COI genes. DNA extracted using Bio-Trace Extraction Kit (Eurx, Gdańsk, Poland) from dissected insect abdomens for up to three individual insects per species (plus negative controls), was used for amplicon library preps following a modified two-step PCR library preparation protocol provided by (69). In the first round of PCR, we amplified two marker regions of interest, using template-specific primers 515F/806R $(70,71)$ and COIBF3//COIBR2 (72) with Illumina adapter tails. The PCR products were purified using SPRI magnetic beads and then used as the template for the second, indexing PCR reaction. Pooled libraries were sequenced on an Illumina MiSeq v3 lane ( $2 \times 300 \mathrm{bp}$ reads) at the Institute of Environmental Sciences of Jagiellonian University. The primer sequences and detailed protocols for amplicon library preparation are provided in Dataset Table S2.

Analyses of amplicon sequencing data. We processed $16 \mathrm{~S}$ rRNA and COI genes amplicon data using mothur v. 1.43.0 (73) following a pipeline detailed in the Supplementary Material. Initially, all amplicon datasets were split into bins corresponding to the two target genes based on primer sequences, and each bin was analyzed separately. For both bins, we assembled forward and reverse reads into contigs, which were then quality-filtered. The contigs were then dereplicated, and the sequences that occurred only once in the dataset (singletons) were discarded. Then, we aligned contigs against the corresponding reference database, removing those that did not align properly. For analysis of 16S rRNA gene sequences, the aligned sequences were screened for chimeric sequences using UCHIME, and then classified by taxonomy. Finally, the sequences were clustered at $97 \%$ identity level using the nearest-neighbor algorithm and divided into OTUs.

\section{Metagenomic library preparation and sequencing}

We sequenced bacteriome metagenomic libraries for three species: C. krueperi (specimen ID: CALKRU), D. multireticulata (DICMUL), and R. scytha (RANSCY). We extracted DNA from dissected bacteriomes of individual insects using a Sherlock AX DNA extraction kit (A\&A Biotechnology, Gdynia, Poland). After fragmentation using Covaris E220 sonificator, we used it for metagenomic library preparation using NEBNext Ultra II DNA Library Prep Kit for Illumina (BioLabs, New England), with the target insert length of $350 \mathrm{bp}$. The library pool, including three target species and other samples, was sequenced on Illumina HiSeq X SBS lane by NGXBio (San Francisco, CA, U.S.A.).

\section{Metagenome characterization and symbiont genome annotation}

Metagenomic reads were filtered by quality scores using 'iu-filter-quality-minoche' program included in illumina-utils software v1.4.4 (74) with default parameters, as described by (75). Contigs were assembled using Megahit v1.2.9 (k-mer 255, min contig size 1000) (76). Because of the known issue of index swapping that occurs during cluster formation and sequencing on Illumina platforms $(77,78)$ which can lead to cross-contamination among samples in multiplexed lanes, we filtered the resulting assemblies for cross-contamination. We discarded all contigs that had $>10 \mathrm{X}$ greater coverage estimated based on strictly mapped reads from another library with overlapping indices than based on reads from the same library, after confirming that most of the removed low-coverage contigs indeed perfectly matched high-coverage microbial genomes in these libraries. 
We identified symbiont contigs using Blastn and tBlastx searches against a custom database containing genomes of multiple known insect symbionts, verifying the identifications using coverage and GC contents information (computed using BBTools v. 38.78). Then, for Sulcia and Vidania contigs, we confirmed their circularity and contiguity by read mapping and visualization on Tablet $\mathrm{v}$. 1.20.12.24 (79) and the presence of overlapping ends. We rearranged the circularized genomes to ensure the same orientation and start position as in those published previously. Arsenophonus, Sodalis and Wolbachia genomes were represented by multiple contigs, and we did not attempt to close them.

The genomic contigs of the three latter symbionts were annotated using prokka v.1.14.6 (80), with the default parameters. For Sulcia and Vidania, prokka but also Interproscan and GhostKOALA annotation attempts left multiple obvious gaps and unannotated or hypothetical proteins, and hence we decided to annotate them using a custom Python script, modified from (27). Annotation was conducted by recursive searches for a manually curated set of alignments of protein-coding, rRNA and ncRNA genes from previously characterized Vidania or Sulcia lineages using HMMER 3.1b2 (81). Any open reading frames of at least 300 nucleotides that had not been annotated by the script were manually searched using hmmer and blastx/tblastx against UniProt and NCBI databases. All genes annotated as "hypothetical" or unannotated were carefully manually compared against the top hits in other microorganisms using blastp (https://blast.ncbi.nlm.nih.gov) and HMMER 3.3 (https://www.ebi.ac.uk/Tools/hmmer), resulting in the discovery of additional genes. Reference-based annotations of rRNA genes were supplemented by rRNA searches using RNAmmer v. 1.2 (82), and tRNA searches using tRNAscanSE v. 1.4 (83). For all genes, we aligned all copies classified as functional using mafft v. 7.221 (84). In case of proteincoding genes, alignments were conducted in protein space and reverse-translated to nucleotide space.

The taxon-annotated GC-coverage plots for symbiont contigs were drawn using $\mathrm{R}$ v. 4.0.2 ( $\mathrm{R}$ Development Core Team) with ggplot2 package (85). Genomes were visualized using DNAPlotter GUI. In order to reconstruct amino acid and B vitamin biosynthetic pathways, we translated circular Sulcia and Vidania genomes as well as Sodalis, Arsenophonus and Wolbachia contigs to amino acids and annotated them against KEGG with GhostKOALA (genus_prokaryotes) (86). After that, the presence of genes involved in biosynthetic pathways was checked manually.

\section{Cloning, amplification, and phylogenetic analyses}

For the four species for which metagenomes were not sequenced (D. pannonica, D. europaea, R. edirneus and $P$. platypus), we obtained full-length $16 \mathrm{~S}$ rRNA gene sequences of symbionts through molecular cloning in Escherichia coli cells as described previously (43). We also PCR-amplified planthopper mitochondrial $C y t B$ and nuclear $18 \mathrm{~S}$ and $28 \mathrm{~S}$ rRNA genes (details in Dataset Table S2). Purified PCR products were Sanger-sequenced by Genomed S.A. (Warsaw, Poland). Trimmed reads were merged into contigs, aligned, and alignments inspected using CodonCode Aligner v. 8.0.2 (CodonCode Corp., Centerville, USA).

We conducted phylogenetic analyses of concatenated insect marker genes and bacterial 16S rRNA genes, including those obtained from metagenomes using MEGA 7 software (87) using Maximum Likelihood algorithm assuming the GTR (for insect genes) and GTR+GAMMA (for bacterial genes) models and with 1000 bootstrap replicates.

\section{Microscopy}

Light (LM) and electron (TEM) microscopy. Partially dissected abdomens of females of each of the seven species were fixed in $2.5 \%$ glutaraldehyde solution in $0.1 \mathrm{M}$ phosphate buffer $(\mathrm{pH} 7.4)$ in the field. In the laboratory, after washing with the same buffer with the addition of sucrose $(5.8 \%)$, they were postfixed in $1 \%$ solution of osmium tetroxide, dehydrated in ethanol and acetone series, and embedded in epoxy resin Epon 812 (SERVA, Heidelberg, Germany). For histological analyses, the resin blocks were cut into serial, semithin sections ( $1 \mu \mathrm{m}$ thick), stained in $1 \%$ methylene blue in $1 \%$ borax, and observed under the Nikon Eclipse 80i light microscope. Ultrathin sections ( $90 \mathrm{~nm}$ thick) for ultrastructural analyses were contrasted with lead citrate and uranyl acetate and observed under the JEOL JEM 2100 electron transmission microscope.

Fluorescence microscopy. For fluorescence in situ hybridization (FISH), insects preserved in $80 \%$ ethanol were rehydrated and postfixed in $4 \%$ paraformaldehyde for 2 hours. Then, the material was dehydrated again in the increasing concentration of ethanol and acetone and embedded in the Technovit 8100 resin (Kulzer, Wehrheim, Germany). Resin blocks were cut into semithin sections (1 $\mu \mathrm{m}$ thick) and hybridized overnight at room temperature with symbiont-specific probes. The probe details and hybridization reaction conditions are provided in Suppl. Table S2. After hybridization, the slides were washed in PBS, dried, covered with ProLong Gold Antifade Reagent (Life Technologies), and examined using a confocal laser scanning microscope Zeiss Axio Observer LSM 710.

\section{Acknowledgments}

We thank John McCutcheon for permission to use the facilities at the University of Montana, advice, and valuable comments, Ada Jankowska, Monika Prus, and Mateusz Buczek for laboratory assistance, Marcin Walczak for 
bioRxiv preprint doi: https://doi.org/10.1101/2021.04.07.438848; this version posted April 9,2021. The copyright holder for this preprint (which was not certified by peer review) is the author/funder, who has granted bioRxiv a license to display the preprint in perpetuity. It is made available under aCC-BY 4.0 International license.

providing D. europaea specimens, Gernot Kunz and Ilia Gjonov for insect images. This project was supported by the Polish National Science Centre grants 2017/26/D/NZ8/00799 (to A.M.) and 2018/30/E/NZ8/00880 (to P.Ł.) as well as Polish National Agency for Academic Exchange grant PPN/PPO/2018/1/00015 (P.t.). The openaccess publication of this article was funded by the Priority Research Area BioSunder the program "Excellence Initiative - Research University" at the Jagiellonian University in Krakow

\section{Supplementary information and data availability}

Supplementary Information file includes nine

Supplementary Figures and the details of amplicon analysis workflow. Supplementary Tables file includes twelve

Supplementary Tables. Accession numbers for all sequencing datasets are listed in Supplementary Table S11.

\section{References}

1. H. Feldhaar, Bacterial symbionts as mediators of ecologically important traits of insect hosts. Ecol. Entomol. 36, 533-543 (2011)

2. K. P. Johnson, et al., Phylogenomics and the evolution of hemipteroid insects. Proc. Natl. Acad. Sci. 115, 12775 (2018).

3. N. A. Moran, J. P. McCutcheon, A. Nakabachi, Genomics and evolution of heritable bacterial symbionts. Annu. Rev. Genet. 42, 165-190 (2008).

4. A. Douglas, How multi-partner endosymbioses function. Nat. Rev. Microbiol. 14, 731-743 (2016).

5. E. Frago, S. E. Zytynska, N. E. Fatouros, "Microbial symbionts of herbivorous species across the insect tree" in Mechanisms Underlying Microbial Symbiosis, (Academic Press Inc., 2020), pp. 111-159.

6. A. E. Douglas, The microbial dimension in insect nutritional ecology. Funct. Ecol. 23, 38-47 (2009).

7. J. McCutcheon, B. R. McDonald, N. Moran, Convergent evolution of metabolic roles in bacterial co-symbionts of insects. Proc. Natl. Acad. Sci. 106, 15394-15399 (2009).

8. J. P. McCutcheon, N. A. Moran, Functional convergence in reduced genomes of bacterial symbionts spanning $200 \mathrm{My}$ of evolution. Genome Biol. Evol. 2, 708-718 (2010).

9. G. M. Bennett, N. A. Moran, Small, smaller, smallest: the origins and evolution of ancient dual symbioses in a phloemfeeding insect. Genome Biol. Evol. 5, 1675-1688 (2013).

10. M. Kobiałka, A. Michalik, M. Walczak, Ł. Junkiert, T. Szklarzewicz, Sulcia symbiont of the leafhopper Macrosteles laevis (Ribaut, 1927) (Insecta, Hemiptera, Cicadellidae: Deltocephalinae) harbors Arsenophonus bacteria. Protoplasma 253, 903-912 (2016).

11. M. Kobiałka, A. Michalik, J. Szwedo, T. Szklarzewicz, Diversity of symbiotic microbiota in Deltocephalinae leafhoppers (Insecta, Hemiptera, Cicadellidae). Arthropod Struct. Dev. 47, 268-278 (2018).

12. R. Koga, G. M. Bennett, J. R. Cryan, N. A. Moran, Evolutionary replacement of obligate symbionts in an ancient and diverse insect lineage. Environ. Microbiol. 15, 2073-2081 (2013).

13. Y. Matsuura, et al., Recurrent symbiont recruitment from fungal parasites in cicadas. Proc. Natl. Acad. Sci. 115, E5970
(2018).

14. C. Simon, et al., Off-target capture data, endosymbiont genes and morphology reveal a relict lineage that is sister to all other singing cicadas. Biol. J. Linn. Soc. 128, 865-886 (2019).

15. P. Buchner, Endosymbiosis of Animals with Plant Microorganisms (Interscience Publishers, 1965).

16. Y. Kikuchi, Endosymbiotic bacteria in insects: Their diversity and culturability. Microbes Environ. 24, 195-204 (2009).

17. T. Szklarzewicz, A. Michalik, "Transovarial Transmission of Symbionts in Insects" in Oocytes: Maternal Information and Functions, M. Kloc, Ed. (Springer International Publishing, 2017), pp. 43-67.

18. M. Mao, G. M. Bennett, Symbiont replacements reset the coevolutionary relationship between insects and their heritable bacteria. ISME J. 14, 1384-1395 (2020).

19. M. Kobiałka, A. Michalik, D. Świerczewski, T. Szklarzewicz, Complex symbiotic systems of two treehopper species: Centrotus cornutus (Linnaeus, 1758) and Gargara genistae (Fabricius, 1775) (Hemiptera: Cicadomorpha: Membracoidea: Membracidae). Protoplasma 257, 819-831 (2020).

20. J. M. Urban, J. R. Cryan, Two ancient bacterial endosymbionts have coevolved with the planthoppers (Insecta: Hemiptera: Fulgoroidea). BMC Evol. Biol. 12, 87 (2012).

21. H. J. Müller, Die Symbiose der Fulgoroiden (Homoptera Cicadina). Zoologica 98, 1-110 (1940a).

22. H. J. Müller, Die Symbiose der Fulgoroiden (HomopteraCicadina). Zoologica 98, 111-220 (1940b).

23. G. M. Bennett, N. A. Moran, Heritable symbiosis: The advantages and perils of an evolutionary rabbit hole. Proc. Natl. Acad. Sci. 112, 10169 (2015).

24. N. A. Moran, P. Tran, N. M. Gerardo, Symbiosis and insect diversification: an ancient symbiont of sap-feeding insects from the bacterial phylum Bacteroidetes. Appl. Environ. Microbiol. 71, 8802 (2005).

25. G. M. Bennett, S. Abbà, M. Kube, C. Marzachì, Complete genome sequences of the obligate symbionts "Candidatus Sulcia muelleri" and " $\mathrm{Ca}$. Nasuia deltocephalinicola" from the pestiferous leafhopper Macrosteles quadripunctulatus (Hemiptera: Cicadellidae). Genome Announc. 4, e01604-15 (2016).

26. F. Husnik, J. P. McCutcheon, Repeated replacement of an intrabacterial symbiont in the tripartite nested mealybug symbiosis. Proc. Natl. Acad. Sci. 113, E5416 (2016).

27. P. Łukasik, et al., Multiple origins of interdependent endosymbiotic complexes in a genus of cicadas. Proc. Natl. Acad. Sci. 115, E226 (2018).

28. A. Nakabachi, J. Piel, I. Malenovský, Y. Hirose, Comparative genomics underlines multiple roles of Profftella, an obligate symbiont of psyllids: providing toxins, vitamins, and carotenoids. Genome Biol. Evol. 12, 19751987 (2020).

29. V. Boscaro, et al., Parallel genome reduction in symbionts descended from closely related free-living bacteria. Nat. Ecol. Evol. 1, 1160-1167 (2017).

30. J. P. McCutcheon, N. A. Moran, Extreme genome reduction in symbiotic bacteria. Nat. Rev. Microbiol. 10, 13-26 (2012).

31. N. A. Moran, G. M. Bennett, The tiniest tiny genomes. Annu. Rev. Microbiol. 68, 195-215 (2014).

32. B. M. Boyd, et al., Two bacterial genera, Sodalis and Rickettsia, associated with the seal louse Proechinophthirus fluctus (Phthiraptera: Anoplura). Appl. Environ. Microbiol. 82, 3185-3197 (2016).

33. E. Nováková, F. Husník, E. Šochová, V. Hypša, Arsenophonus and Sodalis symbionts in louse flies: an analogy to the Wigglesworthia and Sodalis system in tsetse flies. Appl. Environ. Microbiol. 81, 6189 (2015). 
bioRxiv preprint doi: https://doi.org/10.1101/2021.04.07.438848; this version posted April 9, 2021. The copyright holder for this preprint

(which was not certified by peer review) is the author/funder, who has granted bioRxiv a license to display the preprint in perpetuity. It is made available under aCC-BY 4.0 International license.

34. D. Santos-Garcia, et al., To B or Not to B: Comparative genomics suggests Arsenophonus as a source of B vitamins in whiteflies. Front. Microbiol. 9, 2254 (2018).

35. A. L. Clayton, et al., A novel human-infection-derived bacterium provides insights into the evolutionary origins of mutualistic insect-bacterial symbioses. PLoS Genet. 8, e1002990-e1002990 (2012).

36. J. Dittmer, T. Lusseau, X. Foissac, F. Faoro, Skipping the insect vector: Plant stolon transmission of the phytopathogen $\mathrm{Ca}$. Phlomobacter fragaria from the Arsenophonus clade of insect endosymbionts. Insects 12 (2021).

37. E. Nováková, V. Hypša, N. A. Moran, Arsenophonus, an emerging clade of intracellular symbionts with a broad host distribution. BMC Microbiol. 9, 143 (2009).

38. E. Šochová, F. Husník, E. Nováková, A. Halajian, V. Hypša, Arsenophonus and Sodalis replacements shape evolution of symbiosis in louse flies. PeerJ 5, e4099-e4099 (2017).

39. G. P. Taylor, P. C. Coghlin, K. D. Floate, S. J. Perlman, The host range of the male-killing symbiont Arsenophonus nasoniae in filth fly parasitioids. J. Invertebr. Pathol. 106, 371-379 (2011).

40. A. Michalik, W. Jankowska, M. Kot, A. Gołas, T. Szklarzewicz, Symbiosis in the green leafhopper, Cicadella viridis (Hemiptera, Cicadellidae). Association in statu nascendi? Arthropod Struct. Dev. 43, 579-587 (2014).

41. A. Bressan, J. Arneodo, M. Simonato, W. P. Haines, E. Boudon-Padieu, Characterization and evolution of two bacteriome-inhabiting symbionts in cixiid planthoppers (Hemiptera: Fulgoromorpha: Pentastirini). Environ. Microbiol. 11, 3265-3279 (2009).

42. A. Bressan, K. L. Mulligan, Localization and morphological variation of three bacteriome-inhabiting symbionts within a planthopper of the genus Oliarus (Hemiptera: Cixiidae). Environ. Microbiol. Rep. 5, 499-505 (2013).

43. A. Michalik, J. Szwedo, A. Stroiński, D. Świerczewski, T. Szklarzewicz, Symbiotic cornucopia of the monophagous planthopper Ommatidiotus dissimilis (Fallén, 1806) (Hemiptera: Fulgoromorpha: Caliscelidae). Protoplasma 255, 1317-1329 (2018).

44. T. Szklarzewicz, D. Świerczewski, A. Stroiński, A. Michalik, Conservatism and stability of the symbiotic system of the invasive alien treehopper Stictocephala bisonia (Hemiptera, Cicadomorpha, Membracidae). Ecol. Entomol. 45, 876-885 (2020).

45. C. Dale, I. Maudlin, Sodalis gen. nov. and Sodalis glossinidius sp. nov., a microaerophilic secondary endosymbiont of the tsetse fly Glossina morsitans morsitans. Int. J. Syst. Evol. Microbiol. 49, 267-275 (1999).

46. S. M. Kuechler, K. Dettner, S. Kehl, Characterization of an obligate intracellular bacterium in the midgut epithelium of the bulrush bug Chilacis typhae (Heteroptera, Lygaeidae, Artheneinae). Appl. Environ. Microbiol. 77, 2869 (2011).

47. G. M. Bennett, M. Mao, Comparative genomics of a quadripartite symbiosis in a planthopper host reveals the origins and rearranged nutritional responsibilities of anciently diverged bacterial lineages. Environ. Microbiol. 20, 4461-4472 (2018).

48. J. Xue, et al., Genomes of the rice pest brown planthopper and its endosymbionts reveal complex complementary contributions for host adaptation. Genome Biol. 15, 521 (2014).

49. A. C. Darby, et al., Characteristics of the genome of Arsenophonus nasoniae, son-killer bacterium of the wasp Nasonia. Insect Mol. Biol. 19, 75-89 (2010).

50. D. C. Bublitz, et al., Peptidoglycan production by an insectbacterial mosaic. Cell 179, 703-712.e7 (2019).

51. M. A. Campbell, et al., Changes in endosymbiont complexity drive host-level compensatory adaptations in cicadas. $m$ Bio 9, e02104-18 (2018).

52. M. Kupper, C. Stigloher, H. Feldhaar, R. Gross, Distribution of the obligate endosymbiont Blochmannia floridanus and expression analysis of putative immune genes in ovaries of the carpenter ant Camponotus floridanus. Arthropod Struct. Dev. 45, 475-487 (2016).

53. M. Swiatoniowska, A. Ogorzalek, A. Golas, A. Michalik, T. Szklarzewicz, Ultrastructure, distribution, and transovarial transmission of symbiotic microorganisms in Nysius ericae and Nithecus jacobaeae (Heteroptera: Lygaeidae: Orsillinae). Protoplasma 250, 325-332 (2013).

54. T. Szklarzewicz, M. Kalandyk-Kołodziejczyk, K. Michalik, W. Jankowska, A. Michalik, Symbiotic microorganisms in Puto superbus (Leonardi, 1907) (Insecta, Hemiptera, Coccomorpha: Putoidae). Protoplasma 255, 129-138 (2018).

55. S. Aksoy, X. Chen, V. Hypsa, Phylogeny and potential transmission routes of midgut-associated endosymbionts of tsetse (Diptera: Glossinidae). Insect Mol. Biol. 6, 183-190 (1997).

56. C. Dale, G. R. Plague, B. Wang, H. Ochman, N. A. Moran, Type III secretion systems and the evolution of mutualistic endosymbiosis. Proc. Natl. Acad. Sci. 99, 12397 (2002).

57. Y. Guo, et al., Vertical transmission of Wolbachia is associated with host vitellogenin in Laodelphax striatellus. Front. Microbiol. 9, 2016-2016 (2018).

58. J. K. Herren, J. C. Paredes, F. Schüpfer, B. Lemaitre, Vertical transmission of a Drosophila endosymbiont via cooption of the yolk transport and internalization machinery. mBio $\mathbf{4}$, e00532-12 (2013).

59. M. Bright, S. Bulgheresi, A complex journey: transmission of microbial symbionts. Nat. Rev. Microbiol. 8, 218-230 (2010).

60. V. G. Martinson, et al., Multiple origins of obligate nematode and insect symbionts by a clade of bacteria closely related to plant pathogens. Proc. Natl. Acad. Sci. 117, 31979 (2020).

61. S. Enomoto, A. Chari, A. L. Clayton, C. Dale, Quorum sensing attenuates virulence in Sodalis praecaptivus. Cell Host Microbe 21, 629-636.e5 (2017).

62. J. P. McCutcheon, B. M. Boyd, C. Dale, The life of an insect endosymbiont from the cradle to the grave. Curr. Biol. 29, R485-R495 (2019).

63. C. Braendle, et al., Developmental origin and evolution of bacteriocytes in the aphid-Buchnera symbiosis. PLoS Biol. 1, E21 (2003).

64. R. M. Fisher, L. M. Henry, C. K. Cornwallis, E. T. Kiers, S. A. West, The evolution of host-symbiont dependence. Nat. Commun. 8, 15973-15973 (2017).

65. N. A. Moran, Symbiosis as an adaptive process and source of phenotypic complexity. Proc. Natl. Acad. Sci. 104, 8627 (2007).

66. A. Nakabachi, et al., Defensive bacteriome symbiont with a drastically reduced genome. Curr. Biol. 23, 1478-1484 (2013).

67. M. Mao, X. Yang, G. M. Bennett, Evolution of host support for two ancient bacterial symbionts with differentially degraded genomes in a leafhopper host. Proc. Natl. Acad. Sci. 115, E11691 (2018).

68. R. A. Chong, N. A. Moran, Evolutionary loss and replacement of Buchnera, the obligate endosymbiont of aphids. ISME J. 12, 898-908 (2018).

69. T. C. Glenn, Field guide to next-generation DNA sequencers. Mol. Ecol. Resour. 11, 759-769 (2011).

70. A. Apprill, S. McNally, R. Parsons, L. Weber, Minor revision to V4 region SSU rRNA 806R gene primer greatly increases detection of SAR11 bacterioplankton. Aquat. Microb. Ecol. 75, 129-137 (2015).

71. A. E. Parada, D. M. Needham, J. A. Fuhrman, Every base matters: assessing small subunit rRNA primers for marine microbiomes with mock communities, time series and global field samples. Environ. Microbiol. 18, 1403-1414 (2016). 
bioRxiv preprint doi: https://doi.org/10.1101/2021.04.07.438848; this version posted April 9, 2021. The copyright holder for this preprint (which was not certified by peer review) is the author/funder, who has granted bioRxiv a license to display the preprint in perpetuity. It is made available under aCC-BY 4.0 International license.

72. V. Elbrecht, et al., Validation of COI metabarcoding primers for terrestrial arthropods. PeerJ 7, e7745 (2019).

73. P. D. Schloss, et al., Introducing mothur: Open-source, platform-independent, community-supported software for describing and comparing microbial communities. Appl. Environ. Microbiol. 75, 7537 (2009).

74. A. M. Eren, J. H. Vineis, H. G. Morrison, M. L. Sogin, A filtering method to generate high quality short reads using illumina paired-end technology. PLOS ONE 8, e66643 (2013).

75. A. M. Eren, et al., Anvi'o: an advanced analysis and visualization platform for 'omics data. PeerJ 3, e1319 (2015).

76. D. Li, et al., MEGAHIT v1.0: A fast and scalable metagenome assembler driven by advanced methodologies and community practices. Methods 102, 3-11 (2016).

77. M. Costello, et al., Characterization and remediation of sample index swaps by non-redundant dual indexing on massively parallel sequencing platforms. BMC Genomics $\mathbf{1 9}$, 332 (2018)

78. Illumina, Effects of index misassignment on multiplexing and downstream analysis (2018).

79. I. Milne, et al., Using Tablet for visual exploration of secondgeneration sequencing data. Brief. Bioinform. 14, 193-202
(2013).

80. T. Seemann, Prokka: rapid prokaryotic genome annotation. Bioinformatics 30, 2068-2069 (2014).

81. S. R. Eddy, Accelerated profile HMM searches. PLOS Comput. Biol. 7, 1-16 (2011).

82. K. Lagesen, et al., RNAmmer: consistent and rapid annotation of ribosomal RNA genes. Nucleic Acids Res. 35, 3100-3108 (2007).

83. T. M. Lowe, S. R. Eddy, tRNAscan-SE: a program for improved detection of transfer RNA genes in genomic sequence. Nucleic Acids Res. 25, 955-964 (1997).

84. K. Katoh, D. M. Standley, MAFFT multiple sequence alignment software version 7: Improvements in performance and usability. Mol. Biol. Evol. 30, 772-780 (2013).

85. H. Wickham, ggplot2. WIREs Comput. Stat. 3, 180-185 (2011).

86. M. Kanehisa, Y. Sato, K. Morishima, BlastKOALA and GhostKOALA: KEGG tools for functional characterization of genome and metagenome sequences. Comput. Resour. Mol. Biol. 428, 726-731 (2016).

87. S. Kumar, G. Stecher, K. Tamura, MEGA7: molecular evolutionary genetics analysis version 7.0 for bigger datasets. Mol. Biol. Evol. 33, 1870-1874 (2016). 